\title{
Diacronie
}

Studi di Storia Contemporanea

$\mathrm{N}^{\circ} 20,4 \mid 2014$

II diritto militante

\section{La Società Dante Alighieri da Costantinopoli a Istanbul. 1895-1922: diffusione della lingua e pedagogia nazionale}

\section{Stefania De Nardis}

\section{(2) OpenEdition \\ Journals}

Edizione digitale

URL: http://journals.openedition.org/diacronie/1785

DOI: 10.4000/diacronie. 1785

ISSN: 2038-0925

Editore

Association culturelle Diacronie

Notizia bibliografica digitale

Stefania De Nardis, «La Società Dante Alighieri da Costantinopoli a Istanbul. 1895-1922: diffusione della lingua e pedagogia nazionale », Diacronie [Online], № 20, 4 | 2014, documento 14, Messo online il 01 décembre 2014, consultato il 19 avril 2019. URL : http://journals.openedition.org/diacronie/1785 ; DOI : 10.4000/diacronie. 1785 


\section{Diacronie}

\section{La Società Dante Alighieri da Costantinopoli a Istanbul. 1895-1922: diffusione della lingua e pedagogia nazionale}

Stefania DE NARDIS *

La Società Dante Alighieri fu fondata a Roma nel 1889. La sede di Costantinopoli fu inaugurata nel 1895; da allora è stata un centro di aggregazione sociale e culturale per la comunità italiana di Istanbul operando attraverso iniziative quali l'istituzione di scuole, la biblioteca, l'organizzazione di pubbliche conferenze, la promozione della lingua italiana. Ma la Società è stata anche il riflesso dei cambiamenti sociali e politici che da Roma si imponevano alle comunità italiane all'estero. Attraverso le fonti conservate presso l'Archivio della Società, questo articolo intende analizzare da una parte il modo in cui la direzione centrale della Società ha affrontato il passaggio cruciale fra XIV e XX secolo, quando visse il passaggio da un'impostazione basata su ideali risorgimentali a una più marcatamente nazionalista, che sfocerà nella fascistizzazione della Società stessa, dall'altra il modo in cui questa nuova concezione si scontra con il nascente nazionalismo turco alla vigilia del crollo dell'impero.

\section{Italiani a Costantinopoli, «dove costumi e favelle diversissime si incrociano e si sommano in un tumulto discorde», 1895-1911}

Qui ci sono 15 mila italiani. Dove sono?» ${ }^{1}$ si chiedeva Alarico Buonaiuti ${ }^{2}$ in una lettera inviata da Costantinopoli il 18 febbraio 1908 a Giuseppe

\footnotetext{
${ }^{1}$ Archivio Storico Dante Alighieri (da qui, ASDA), fasc. Costantinopoli 1895-1921, 167A. La cifra indicata da Buonaiuti non corrisponde probabilmente con esattezza alla realtà, e lo stesso
} 
Zaccagnini, già presidente del comitato locale della Società Dante Alighieri, divenuto poi segretario generale della società presso la sede centrale di Roma.

Nella storia del comitato di Istanbul della Società Dante Alighieri lo sforzo dei dirigenti locali per implementare l'adesione dei connazionali fu un tratto costante fin dall'atto di fondazione, il 20 settembre $1895^{3}$, un'esigenza ovviamente insita nei fini della Società, ma rafforzata anche dalle direttive societarie centrali, che vedevano da tempo nella comunità italiana della città una delle piazze dalle maggiori potenzialità per gli scopi della Dante.

Già durante i lavori del $\mathrm{IV}^{\circ}$ Congresso generale di Firenze del novembre 1893, infatti, i territori del Levante erano stati confermati di prioritario interesse, in linea con una tendenza inaugurata proprio a partire da fine Ottocento, con lo sviluppo dei comitati esteri, e concorde con la visione espansionistica crispina.

L'idea di creare un comitato a Istanbul trovò in questo clima un terreno fertilissimo. Nella capitale ottomana meglio che altrove poteva attecchire il Leitmotiv del ritornare ad antiche grandezze. La decisione fu presa

\begin{abstract}
in una giornata dell'Agosto del 1895, fra una schiera di giovani riuniti a banchetto nell'incantevole altura di Giamligià [Çamlıca]. La mole altera della torre di Galata, e laggiù, nello sfondo cupo del mar Nero, i ruderi del Castello dei Genovesi, parlavano loro dell'antica grandezza d'Italia, mentre nell'azzurro denso del Bosforo, dallo Stazionario delle navi mercantili sventolava al sole il tricolore della patria risorta, anelante a nuovi destini4.
\end{abstract}

\footnotetext{
Zaccagnini in una sua pubblicazione del 1909 indica una cifra di circa 8.000 italiani regolarmente registrati in città, cfr. ZACCAGNINI, Giuseppe, La vita a Costantinopoli, Torino, F.lli Bocca, 1909, p. 71. Angiolo Mori - cui si deve uno dei primi e più completi studi sulla comunità italiana di Istanbul, nel 1906 - premettendo che non era possibile risalire al numero esatto poiché non tutti i residenti italiani a Istanbul si iscrissero ai registri consolari, afferma che «mentre i nuovi registri consolari, aperti il $1^{\circ}$ gennaio 1881, danno al 31 dicembre 1905 un numero di iscritti di 8922 nazionali, i calcoli più recenti farebbero ammontare la nostra colonia a 12.500 per alcuni, a 14.000 nazionali per gli altri», MORI, Angiolo, Gli italiani a Costantinopoli. Monografia coloniale presentata alla Camera di Commercio italiana di Costantinopoli alla mostra degli italiani all'estero, Modena, Società tipografica modenese, 1906, p. 210.

${ }^{2}$ Fratello di Ernesto, storico e sacerdote esponente del modernismo.

3 Secondo quanto si può dedurre dalle relazioni inviate alla sede centrale e, dal 1920 al 1925, dal Bollettino ufficiale della Dante di Istanbul, il numero di soci iscritti per anno fu: 22 al momento della fondazione, nel 1895; 102 nel 1896; 150 nel 1897; 210 nel 1898; 225 nel 1899; 283 nel 1908, 269 nel 1909; 168 nel 1918; circa 600 nel 1919; 1206 nel 1922; 1258 nel 1923; 1197 nel 1924; 1102 nel 1925; meno di 900 nel 1931, circa 1000 nel 1938.

4 MORI, Angiolo, Gli italiani a Costantinopoli, cit., p. 269.
} 
I giovani in questione erano alcuni membri della Società operaia di mutuo soccorso 5 e la nascita del comitato di Istanbul coincise con una fase di svolta che la Dante stava avviando a livello nazionale rispetto all'impostazione che si era data nel 1889 , anno della sua fondazione ${ }^{6}$.

Con lo sviluppo dei primi comitati esteri, essa ridefinì le sue finalità passando da un programma ispirato all'esigenza di «preparare l'annessione di territori irredenti all'Italia in piena Triplice Alleanza» 7 , a una visione più ampia e meglio strutturata, una missione che si estese alle comunità degli emigrati ovunque esse si trovassero, e segnatamente a quelle residenti in territori di più spiccato interesse politico ed economico per l'Italia. L'uomo più rappresentativo di questa virata fu il senatore Pasquale Villari ${ }^{8}$. Il nuovo orientamento prevedeva di affiancare all'originario mandato che la Dante si era imposta - la diffusione della lingua italiana - un programma di matrice più pedagogica che avrebbe dovuto fare leva sulle istituzioni scolastiche e educative: biblioteche, corsi di lingua italiana, conferenze, serate musicali e iniziative culturali in genere.

Fin dai primi anni della sua esistenza il comitato di Costantinopoli, inaugurato in occasione del XXV anniversario della liberazione di Roma, fece registrare un'intensa attività tesa a perseguire fedelmente questa linea programmatica. Nel primo

5 «Nel vasto Lavorio di propaganda di assimilazione che si fa in Oriente da tutte le nazioni europee, nella lotta aspra ed incessante che si combatte per l'incremento politico e commerciale, il predominio della lingua ha evidentemente una importanza massima. Gli è perciò che a dare sempre più vasto sviluppo al patrio idioma, sorse, in seno alla Società Operaia l'idea di fondare un comitato della Dante Alighieri». GOSLINO, Pietro, PROVIDENTI, Ferdinando, Società Operaia di Mutuo Soccorso in Costantinopoli. Memoria storica (1863-1906), Costantinopoli, Tipografia Ferd. Walla, 1906, p. 138. Nonostante questo rapporto di filiazione con il trascorrere degli anni, come si vedrà più avanti, le relazioni fra le due «consorelle» furono caratterizzati da diversi momenti di tensione, che si acuiranno nel periodo fascista, quando le fratture politiche all'interno della comunità si riversarono inevitabilmente nelle sedi dell'associazionismo italiano. ${ }^{6}$ Sulla storia della Società si vedano: BARBERA, Piero, La Dante Alighieri. Relazione storica al XXV congresso Trieste-Trento 1919, Roma, Società Nazionale Dante Alighieri, 1919; SCODNIK, Enrico, «La Società Nazionale Dante Alighieri nei suoi primi anni di vita», in La Rivista Dalmatica, 1-4/1966; CAPARELLI, Filippo, La «Dante Alighieri», Roma, Bonacci, 1987; SALVETTI, Patrizia, Immagine nazionale ed emigrazione nella Società «Dante Alighieri», Roma, Bonacci, 1995; PISA, Beatrice, Nazione e politica nella Società «Dante Alighieri», Roma Bonacci, 1995.

${ }^{7}$ SALVETTI, Patrizia, op. cit., p. 16.

8 Ibidem, pp. 31 et seq. Villari fu presidente della Dante dal 1896 al 1901. La rinnovata impostazione che Villari diede alla Società era perfettamente in linea con la sua formazione di storico meridionalista e di uomo del Risorgimento. Sulla sua figura si vedano MORETTI, Mauro, Pasquale Villari, storico e politico, Napoli, Liguori, 2005, p. 157; BALDASSERONI, Francesco, Pasquale Villari. Profilo biografico e bibliografia degli scritti, Firenze, Galileiana, 1907; SALVEMINI, Gaetano, Pasquale Villari, in ID., Scritti vari (1900-1957), Milano, Feltrinelli, 1978, pp. 57-8o. Sulla sua presidenza alla Dante in rapporto all'irredentismo trentino cfr. MONTELEONE, Renato (a cura di), Dai carteggi di Pasquale Villari: la Società "Dante Alighieri" e l'attività nazionale nel Trentino (1896-1916), Trento, Istituto per la storia del Risorgimento italiano, 1963. 
quinquennio il numero di soci crebbe dai ventidue fondatori ai 225 del 1899; purtroppo la documentazione prodotta dal comitato è scarsa e lacunosa, ma dai pochi fogli relativi ai primi mesi dalla fondazione si possono già avere indicazioni interessanti sulla colonia e sul suo livello di conoscenza, qualitativa e quantitativa, della lingua e della cultura italiane, e sulla funzione di queste ultime nella riflessione sul nesso fra cultura e idea nazionale9.

Le relazioni inviate a Roma dai presidenti locali evidenziano soprattutto le due consuete questioni che riguardarono quanti operavano per il mantenimento e lo sviluppo dell'italianità della colonia, e che avrebbero analogamente caratterizzato il primo decennio di esistenza della Dante, vale a dire il rapporto con le numerosissime scuole congregazioniste presenti in città e la concorrenza con le associazioni culturali francesi che avevano rispettivi, analoghi scopi. Fra queste ultime la più acerrima concorrente fu l'Alliance Française.

A questo si aggiungeva, elemento comune a tutti i comitati della Dante, una pressoché continua richiesta di fondi, indirizzata direttamente alla sede centrale o al ministero degli Affari esteri ${ }^{10}$. Ma le relazioni non mancano di mostrare anche uno spaccato sul clima interno alla comunità dei soci, sui legami fra questa e le autorità diplomatiche italiane e, infine, specie nei momenti di maggiore tensione dovuti alle crisi internazionali che coinvolsero Italia e Turchia, i complessi rapporti con le autorità locali ${ }^{11}$.

Se a livello nazionale, all'influente personalità del senatore Pasquale Villari seguirono Luigi Rava (1903-1906) - ministro dell'Agricoltura nel secondo governo Giolitti e poi dell'istruzione nel terzo - e il senatore Paolo Boselli con la sua trentennale presidenza conclusasi nel 1932; il periodo che va dalla fondazione al 1911 vide susseguirsi sette presidenti alla guida del comitato ${ }^{12}$. I primi due anni di vita della Dante ad Istanbul furono affidati alla presidenza del prof. Federico Morandi ${ }^{13}$. Dalla sua corrispondenza con la sede centrale si rileva che il numero dei soci già due mesi

9 Cfr. PISA, Beatrice, op. cit.

${ }^{10}$ SALVETTI, Patrizia, op. cit., p. 25.

${ }^{11}$ In generale sulla comunità italiana di Istanbul si vedano PANNUTI, Alessandro, La comunità italiana di Istanbul nel XX secolo. Ambiente e persone, Isis, Istanbul 2006; DE GASPERIS, Attilio, FERRAZZA, Roberta (a cura di), Gli italiani di Istanbul. Figure, comunità e istituzioni dalle riforme alla repubblica (1839-1923), Torino, Centro altreitalie, 2007.

${ }_{12}$ Dal 1895 al 1945 furono diciassette i presidenti della società: Giuseppe Zaccagnini (18951900); Angelo Zanotti (1900-1901); Lewis F. Mizzi, (1901-1907); dal 1907 al 1910 C. D’Agostino, Alberto Theodoli, Edoardo De Nari; dal 1910 al 1915 Riccardo Zeri e Aldo Lombardo; Luigi Joli (1920-1930); Aldo Mei (1930-1934); Carlo Rocco Simen (1934-1935); Francesco Feliziani (19351936); Giulio Jacopi (1936-1938); Lamberto Biancone (1938-1940); Ezio Bartalini (1940-1945). 13 I primi presidenti, soci della Società operaia, erano quasi sempre insegnanti presso le scuole italiane. Di queste purtroppo molta documentazione precedente la grande guerra è andata smarrita a causa di un incendio. 
dopo l'inaugurazione della sede - stabilita provvisoriamente presso la scuola tecnicocommerciale italiana Principe Amedeo - «supera la sessantina», che si sarebbero tenute tre conferenze per i soci e che dal gennaio successivo si sarebbe pubblicata una rivista quindicinale per iniziativa della Camera di commercio, «che sarà anche organo ufficiale di questo comitato» ${ }^{14}$. Si tratta de «La Rassegna Italiana», rivista nata, di fatto, dall'accordo fra le due società ma che due anni dopo sarebbe rimasta organo ufficiale esclusivamente della Camera di commercio' ${ }^{15}$, mentre la Dante avrebbe visto la pubblicazione del suo primo Bollettino ufficiale solo nel 1920.

La corrispondenza di questo primo periodo di attività si sofferma spesso sul rapporto del comitato con gli ambienti cattolici presenti e operanti in città. Non di rado i soci influenti agirono da mediatori fra la Roma dei ministri e quella del papa, durante gli anni della questione romana. Ne è un esempio la vicenda di padre Aurelio Palmieri' ${ }^{16}$, appartenente all'ordine degli Agostiniani dell'Assunzione. Il 17 dicembre 1895 il presidente Morandi inviò una lettera alla presidenza centrale - che dopo la morte di Ruggero Bonghi, nell'ottobre del 1895, fu retta per un breve periodo da Ernesto Nathan - in cui esprimeva le sue opinioni in merito all'agostiniano. Qualche tempo prima, infatti, padre Palmieri - missionario a Istanbul, nel quartiere di Kumkapı - aveva scritto sia alla presidenza nazionale sia a quella di Istanbul richiedendo libri e riviste italiane, testi di Manzoni, De Amicis, Stoppani e copie de «La Nuova Antologia». L'agostiniano così concludeva la sua lettera a Morandi: «sappia che nel clero italiano l'amor della Patria è sempre vivo, sempre fecondo, e da per tutto malgrado le influenze politiche che sembrano schiacciarci di giorno in giorno, noi inalberiamo il vessillo

\footnotetext{
14 ASDA, fasc. Costantinopoli 1895-1921, 167A.

15 Quanto successe viene spiegato in una relazione inviata alla sede centrale nel settembre 1897 dal presidente Giuseppe Zaccagnini: «Nel primo anno la Dante Alighieri pensò subito all'utilità di due cose: avere un giornale italiano a cercar di riallacciare [...] le sparse vestigia della colonia promovendo delle riunioni e per mezzo di letture o di conferenze su svariati argomenti. Il giornale sorto dal vecchio organo della nostra camera di commercio s’intitolò Rassegna Italiana. Tre giovani volenterosi e valorosi che meritano tutti gli elogi e molta gratitudine, l'avv. Giorgio Chabert, l'avv. Alberto Vuccino e il dott. Luigi Mongeri, ne assunsero a tutto loro rischio la pubblicazione avendo redattori principali tutti i componenti il consiglio direttivo del comitato della nostra società. Il giornale così rinnovato, in grande formato in 16 e 32 pagine, uscì puntualmente due volte il mese per tutto un anno. Finito l'anno gli assuntori, per qualche divergenza di criteri con la Camera di commercio, restituirono la Rassegna, secondo i patti stabiliti colla camera stessa, restando perdenti di una somma non indifferente. Sarebbe troppo lungo, e inutile, indagare qui la causa dello insuccesso: ché insuccesso, economicamente almeno, la pubblicazione fu. Però restò l'iniziativa che, seguita, non può che dare risultati degni di non essere abbandonati», ASDA, fasc. Costantinopoli 1895-1921, 167A. Un'analisi dei temi della rivista è in PANNUTI, Alessandro, op. cit., pp. 119-152.

${ }_{16}$ Padre Palmieri fu inoltre autore di una cronaca dell'“Associazione commerciale artigiana di pietà in Costantinopoli”, PALMIERI, Aurelio, Associazione Commerciale Artigiana di pietà in Costantinopoli. Cenni storici 1837-1902, Napoli, Giannini\&figli, 1902. Cfr. PANNUTI, Alessandro, op. cit., pp. 110-114.
} 
dell'italianità»17. Morandi, invitato dalla presidenza generale a esporre il suo parere in merito all'opportunità di esaudire le richieste di Padre Palmieri, rispondeva senza indugio che

la sua richiesta di libri italiani non solo merita di essere soddisfatta, ma io, a nome di questo comitato, la raccomando vivamente [...] perché egli ci sarà di sommo aiuto alla nostra propaganda. Sappia che il villaggio di Cadichioi (Calcedonia) e tutta la costa asiatica sotto Scutari (Haider-pascià, Moda, Fanaraki ecc.) formavano, ancora pochi anni fa, quasi un'isola italiana, la parrocchia era italiana, italiani i preti, ed italiane le principali famiglie che potevano influire sulla gente che va aumentando ogni giorno su quelle ridenti spiagge. Ma dacché vi si stabilirono i preti e le suore francesi, aiutati dalla Santa Sede, qui rappresentata dal Legato apostolico Mons. Bonetti18 (un piemontese!) e ancor più dall'Alliance Française, che qui spende somme favolose, mandando gratuitamente casse di libri, i poveri pochi italiani dovettero ritirarsi, cedere la parrocchia e la chiesa (costruita con denari italiani!) e non fiatare, se non volevano incorrere in disgrazia dei loro superiori. Il padre Aurelio, col quale ho parlato lungamente, è uno dei pochi che abbia resistito, e ci può essere davvero utilissimo, poiché non avendo residenza fissa conosce e frequenta, per i bisogni del suo ministero, la massima parte delle famiglie di Stanbul, di Cadikioi e di Fenerbagè ${ }^{19}$.

Due anni dopo, da Roma si invitava nuovamente la presidenza di Istanbul a inviare notizie su padre Palmieri in seguito a una sua nuova richiesta di volumi. Questa volta il presidente Zaccagnini fu ancora più esplicito nel riferire al segretario generale Donato Sanminiatelli quale sarebbe dovuto essere, secondo la sua opinione, il tipo di rapporto che si sarebbe dovuto istaurare con i religiosi italiani presenti in città:

Il padre Palmieri è persona ottima e un bravo italiano: quindi confermiamo le notizie già altre volte date e preghiamo il consiglio di mandargli i libri che chiede. Il parroco di Sant'Antonio, Padre Caneve [Superiore dei Conventuali], ci chiede per la sua scuola: una carta dell'Europa, una carta dell'Italia e una carta mappamondo. Preghiamo vivamente di compiacere anche lui, che, insieme con altri padri della

\footnotetext{
${ }_{17}$ ASDA, fasc. Costantinopoli 1895-1921, 167A.

${ }_{18}$ A Mons. Augusto Bonetti, Delegato Apostolico a Istanbul dal 24 giugno 1887 fino alla morte, nel 1904, si deve la fondazione a Istanbul dei due istituti dei Salesiani e delle Suore Carmelitane. Alla sua morte lasciò ai Salesiani della città la somma che permise loro di consolidare e garantire il futuro della scuola di arti e mestieri. Cfr. BISKUPSKI, Ludwik, L'origine et l'historique de la Représentation officielle du Saint-Siège en Turquie (1204-1967), Istanbul, Ümit Basımevi, 1968, pp. 81-83; MORI, Angiolo, op. cit., p. 254.

19 ASDA, fasc. Costantinopoli 1895-1921, 167A.
} 
parrocchia è anche socio del nostro comitato e che può, amico, esserci assai utile e, indifferente o ostile, nuocerci o, almeno, non giovarci. Bisogna che anche noi, una volta o l'altra, ci decidiamo, sull'esempio dei francesi, a servirci dell'opera del clero $^{20}$.

I propositi di Zaccagnini erano in sintonia con quelli della presidenza generale - a propria volta in linea con gli indirizzi politici del governo Di Rudinì, di cui Villari era stato ministro dell'istruzione nel primo governo del 1891-92 - e l'impegno profuso da Villari per una nuova impostazione della Dante, più attenta agli aspetti sociali delle comunità italiane e non solo a quelli prevalentemente scolastici, e per caratterizzare la Dante come società, oltre che apolitica, laica ma distante da posizioni anticlericali ${ }^{21}$, trovò in Zaccagnini un buon interlocutore e un valido esecutore.

È stato sottolineato che con la presidenza Villari si istituì fra la Dante e l'istituzione statale «un rapporto dialettico e cooperativo che non vedrà mai la Dante in posizione anti-istituzionale ${ }^{22}$, una presa di posizione che, pur essendosi definita proprio durante gli anni del delicato passaggio dal governo Crispi a quello Di Rudinì, in cui il ridimensionamento della prospettiva espansionistica crispina ebbe fra i suoi primi effetti la soppressione, nel 1891, di circa cinquanta scuole governative nel Levante ${ }^{23}$, fu sostanzialmente seguita anche dal comitato di Istanbul senza far registrare grosse incomprensioni, almeno fino agli anni del fascismo.

Se a Roma la Società si rapportava agevolmente con le autorità governative centrali, e soprattutto con il ministero degli affari esteri, deputato a gestire le questioni

\section{${ }^{20}$ Ibidem.}

${ }^{21}$ SALVETTI, Patrizia, op. cit., p. 34.

22 Ibidem, p. 17.

${ }^{23}$ Giuseppe Lombardo-Radice, in un polemico volume sulla «indecorosa politica della Consulta da Rudinì a Tittoni» - come tuona il sottotitolo - applicata alla gestione delle scuole italiana all'estero, così sintetizza la situazione del decennio 1888-98: «Fu quello il periodo dell'entusiasmo [dopo la fondazione delle scuole governative, nel 1888], a cui seguì ben presto quello dell'insania, poiché quando i germi gettati cominciavano a dare i loro buoni frutti e le scuole andavano sempre più acquistando credito presso le popolazioni, il Ministero Rudinì, nel 1891, per ragioni di gretta economia, procedette alla chiusura di oltre 50 scuole, alcune delle quali popolatissime. Una passeggera fioritura si ebbe nel 1894 con il ritorno di Crispi al potere, ma fu di breve durata; dal 1895 in poi si ebbe il periodo della decadenza [...]. Furono i 14 anni di dominio assoluto e senza controllo del commendatore Scalabrini, ispettore generale, durante i quali la scuola di stato decadde, intisichì, intristì; sulle sue rovine cominciò a spuntare o rifiorire la scuola confessionale... sussidiata e favorita dallo Stato, e contro le scuole di stato e le stesse scuole confessionali italiane ebbero a poco a poco ragione le scuole fondate da altre nazioni, che assorbirono in pochi anni migliaia e migliaia dei nostri alunni [...]», LOMBARDO-RADICE, Giuseppe, Le scuole italiane all'estero. Note sulla indecorosa politica della Consulta da Rudinì a Tittoni, Ortona a mare, Vincenzo Bonanni Editore, 1910. Sulla soppressione degli istituti attuata dal governo Di Rudinì si vedano anche: Relazione sulle scuole italiane all'estero, A.P., Camera dei Deputati, Documenti, Ses. 1890-92, Seduta del 9 marzo 1892. CORSI, Carlo, La soppressione delle scuole italiane in Levante, Venezia, s.e., 1893. 
d'emigrazione, i comitati locali avrebbero dovuto trovare i corrispettivi interlocutori nei rappresentanti del governo presso le colonie, vale a dire nel corpo diplomatico italiano. Le relazioni suggeriscono, invece, un rapporto che nei primi anni di vita della Dante non fu sempre piano con il console e l'ambasciatore italiani. Più volte si sollecitò la direzione centrale affinché "facesse pratiche" perché i diplomatici italiani partecipassero più attivamente alle attività promosse dalla società.

Avvocato, insegnante e direttore della scuola tecnico-commerciale ${ }^{24}$, Giuseppe Zaccagnini fu una delle personalità di maggior rilievo all'interno della comunità italiana di inizio secolo. Il suo nome è noto soprattutto come autore dei due volumi, La vita a Costantinopoli, del 1909, e la riedizione con aggiornamenti dello stesso, nel 1926, Ricordi di Costantinopoli ${ }^{25}$. Poco nota invece la sua attività alla presidenza del comitato locale della Dante, che, seppure durata solo un triennio, dal 1897 al 1899, si distinse per un'intensa operosità che si sarebbe protratta anche al suo rientro in Italia, dove sempre presso la Dante ricoprì la carica di segretario generale dal 1907 al 1926, posizione che gli permise di continuare ad avere un rapporto diretto e intenso con i presidenti suoi successori a Istanbul. Così Zaccagnini descriveva la situazione linguistica e culturale della colonia nel 1897, all’inizio della sua presidenza:

\footnotetext{
Oggi, è vergogna, dirlo, la massima parte degli Italiani che figurano iscritti nei registri del nostro R. consolato, non sanno l’italiano. La loro lingua è il greco o il francese e, se qualcuno, raramente, se ne vergogna, molti ritengono naturalissima la cosa. Però un risveglio, piccolo se si vuole, ma positivo ci fu quando vennero istituite scuole italiane governative; e oggi, moltissimi si lamentano d'essere stati lasciati per il passato privi di mezzo d'istruirsi nella loro lingua e, specialmente tra le signore, non poche replicatamente manifestarono vivissimo il desiderio dell'istituzione d'un corso di studi superiore a quello delle scuole elementari che appunto noi vorremmo istituire ${ }^{26}$.
}

Alle sue riflessioni lucide e scevre da retorica nazionalista, corrispose un programma altrettanto concreto: intensificare i rapporti fra la Dante e le autorità diplomatiche italiane sul posto - «non già perché si reputi necessario alla vita prospera della "Dante Alighieri” il sigillo della ufficialità, ma perché, all'estero specialmente, dove gli equivoci sono frequenti, e non sempre si hanno pronti e facili i mezzi per dissiparli, il sapere consenzienti e quasi collaborativi in un'opera, quelli che

\footnotetext{
24 MORI, Angiolo, op. cit., p. 252; PANNUTI, Alessandro, op. cit., p. 74-79.

25 ZACCAGNINI, Giuseppe, Ricordi di Costantinopoli, Lucchetti, Cingoli 1926.

${ }^{26}$ ASDA, fasc. Costantinopoli 1895-1921, 167A.
} 
rappresentano il paese, significa per lo meno risparmiare molto tempo e molto lavoro» 27 -; ampliare la cerchia dei soci cercandoli, sull'esempio dell'Alliance Française, anche fra gli stranieri; fare leva soprattutto sulle scuole, anche quelle straniere e religiose, per la diffusione della lingua; «se l'Alliance Français, da una colonia che è appena la quinta parte, per numero, della nostra, trova mezzi per sussidiare largamente istituti e diffondere la sua lingua e francesizzare completamente l'ambiente, non c'è ragione, pur riconoscendo per forza la nostra inferiorità in molte cose, per cui anche noi con costanza e buon volere non si debba arrivare a qualche pratico risultato» ${ }^{28}$.

Nel corso del 1898 si finanziarono corsi di italiano nel collegio dei Mechitaristi di Vienna, a Pera, nella scuola parrocchiale di Pangalti diretta dai Frères des écoles chrétiennes, nella scuola dei «padri Giorgiani» di Feryköy, si distribuirono inoltre libri in italiano agli allievi meritevoli della «scuola elleno-cattolica Syphnia» e della scuola parrocchiale dei Conventuali di Sant'Antonio. Fu poi stanziato un sussidio destinato a una scuola di assunzionisti francesi a Zonguldak, importante centro di estrazione carbonifera sul Mar Nero, perché si rendesse obbligatorio l’insegnamento dell'italiano, vista la forte presenza di operai italiani in quella zona ${ }^{29}$. Vennero istituiti due corsi per le «signore e signorine italiane e straniere residenti a Costantinopoli» tenuti da Ernesto Nelli, professore presso la scuola tecnico-commerciale, e dal prof. Ortensio Puorto. I due corsi, uno di livello più avanzato e l'altro destinato a quante ignorassero completamente la lingua, furono frequentati rispettivamente da quattordici e da oltre trenta allieve di varia nazionalità, greca, armena, inglese, tedesca, francese e austriaca. Lo stesso Nelli scriveva al segretario generale, Arturo Galanti, in novembre:

\begin{abstract}
Dalla relazione sul mio corso estivo che ti ho inviato, si rileva che 42 sopra $\mathrm{i}$ cinquanta iscritti erano non italiani che vennero ad imparare l'italiano l'insegnamento del quale era gratuito e obbligatorio; 20 appartenevano ad altre scuole (turche, greche, armene, ebree); 18 alla preparatoria nostra che è il vivaio della scuola secondaria; perché in essa sono accolti gli alunni che hanno già fatto qualche anno di studio in altra scuola e vi rimangono finché, imparato l'italiano, entrano in uno dei quattro corsi nostri. [...] Mi pare che nessuna impresa si possa
\end{abstract}

\footnotetext{
27 Ibidem.

28 Ibidem.

29 Cfr. QUATAERT, Donald, Miners and the State in the Ottoman Empire. The Zonguldak Coalfield. 1822-1920, Oxford, Berghahn Books, 2006.
} 
fare all'estero che più e meglio e tutt'intera entri nel programma della Dante Alighieri ${ }^{30}$.

Dopo le dimissioni di Zaccagnini, l'11 aprile 1900 fu eletto il nuovo consiglio direttivo e Angelo Zanotti ne assunse la presidenza, che avrebbe mantenuto solo per un anno. Giuseppe Zaccagnini lasciava al suo successore 225 iscritti e una riflessione amara, scritta nella sua ultima relazione spedita a Villari il 20 agosto 1899: «sarebbe veramente da disperare del nostro patriottismo se, quale indice, se ne dovesse prendere il rigoglio che la Dante sorta sotto sì lieti auspici e con così alti propositi, ha raggiunto da noi dopo vari anni di vita»31.

Zanotti fece suo questo quadro poco incoraggiante attribuendo le deficienze della Dante prevalentemente alle condizioni economiche della colonia - «la nostra colonia è così fatta che pochissimi sono gli aventi, moltissimi i poveri» -, alla quale le già molte società di beneficenza chiedevano sacrifici economici, e chiudeva una delle sue lettere a Roma invocando l’impegno dell'Ambasciatore italiano almeno per accrescere il numero dei soci - «Studieremo, non di meno di fare qualche cosa, ma abbiamo poca fiducia. L'esperienza insegna che da queste parti l'uovo di una buona idea non schiude se non è covato dalla gallina governativa» - e ricordando come, nonostante le difficoltà, l'impegno della Dante aveva portato risultati concreti nell'adempiere al suo scopo di diffusione della lingua italiana all'interno della società di Istanbul: «qui l'opera della Dante è interessantissima. Da che questo Comitato esiste, circa 150 alunni di nazionalità straniere, ogni anno, in collegi e scuole stranieri, imparano l’italiano» 32 .

\section{La stampa e la scuola}

Dall'aprile del 1901 al settembre del 1907 la presidenza della Dante fu retta da Lewis Mizzi33, anch'egli avvocato a Istanbul e, per la prima volta dalla fondazione del comitato, un presidente di nazionalità non italiana. Lewis era infatti maltese, figlio del magistrato Francesco Mizzi, fratello minore di Fortunato Mizzi34 - fondatore del

\footnotetext{
${ }^{30}$ ASDA, fasc. Costantinopoli 1895-1921, 167A.

${ }^{31}$ Ibidem.

32 Ibidem.

33 Lewis Mizzi fu una delle figure più poliedriche presenti a Istanbul nel quarantennio a cavallo fra il XIX e il XX secolo. Avvocato, si trasferì a Istanbul negli anni Settanta, cfr. SCHIAVONE, Michael J., SCERRI, Louis J., Maltese Biographies of the Twentieth Century, Pietà, Pubblikazzjonijiet Indipendenza, 1997.

34 Su Fortunato Mizzi cfr.: VOLPE, Gioacchino, Italia moderna, Firenze, Sansoni, 1973, p. 209; MEYNIER, Gilbert, RUSSO, Maurizio, L'Europe et la Méditerranée. Stratégies et itinéraires
} 
movimento antiriformista e figura di spicco nella lotta contro l'amministrazione inglese per la difesa della supremazia della lingua italiana nell'istruzione e del cattolicesimo a Malta35. La storia della famiglia Mizzi non è solo la storia del loro impegno politico, ma anche quella di una dinastia di editori e giornalisti. A Malta, fino alla vigilia della seconda guerra il giornale nazionalista «Malta», fondato proprio da Fortunato Mizzi nel 1883, fu l'ultimo baluardo della stampa politica italofona ${ }^{36}$. Presidente della Dante, Lewis Mizzi proseguì dunque a Istanbul l'azione familiare di diffusione della lingua italiana, si trovò però nella curiosa situazione di presiedere il sodalizio deputato a questo scopo essendo contemporaneamente proprietario di un giornale inglese, il «Levant Herald».

Fra gli strumenti più efficaci ai fini della diffusione della lingua, la stampa italiana fu invece una nota dolente all'interno della comunità nel XIX e XX secolo. Nonostante fosse unanimemente riconosciuta dai notabili della colonia l'opportunità e anzi l'esigenza di promuovere la creazione di un giornale italiano, non si arrivò mai a dare vita una testata che potesse eguagliare o almeno tentare di concorrere con quelle in lingua francese e inglese37. A voler indagare le cause di questa carenza - che, considerati la consistenza numerica della comunità e i proclami governativi di quegli anni, tutti tesi a promuovere azioni di diffusione culturale, appare quanto meno singolare - essa non sembra potersi attribuire alla totale mancanza di personalità in grado di portare avanti una simile iniziativa. Almeno tre testate a Istanbul infatti, il francese «Beyoğlu», dell'italo-levantino Gilberto Primi, «La Turquie», bilingue italofrancese e l'inglese «Levant Herald», erano di proprietà di italiani, o, come nel caso di Mizzi, di italofoni sensibili alla causa della diffusione della lingua. La «Rassegna Italiana», come accennato sopra, nonostante le iniziali ambizioni della Dante divenne presto organo ufficiale esclusivamente della Camera di commercio, rimanendo di fatto

politiques et culturels en Méditerranée France et Italie. XIXe-XXe siècles, une approche comparative, Paris, L'Harmattan, 1999, p. 143; CASSAR, Carmel, Society, Culture and Identity in Early Modern Malta, London, Mireva, 2000, p. XXIX; GOODWIN, Stefan, Malta, Mediterranean Bridge, Westport, Greenwood Publishing Group, 2002, p. 87. La figura di Mizzi è ricordata inoltre in due discorsi di commemorazione: CORTIS, Giulio, Fortunato Mizzi, padre della patria. Discorso commemorativo pronunziato nell'aula magna della $R$. Università di Malta il 20 maggio 1922, La Valletta, Tipografia del Malta, 1922; SAMMUT, Giovanni, Orazione ad esaltazione di Fortunato Mizzi, padre della Patria, nel 3o. annuale della sua morte, pronunziata nel Circolo La Giovine Malta, il 18 maggio 1935, La Valletta, Editrice Melitense, 1935.

35 Cfr. DOBIE, Edith, «Malta and her Place in the Commonwealth», in The Western Political Quarterly, 9, 4/1956, pp. 873-883.

${ }_{36}$ PORTELLI, Sergio, op. cit., p. 344.

37 Cfr. GROC, Gérard, ÇAĞLAR, İbrahim, La presse française de Turquie de 1795 à nos jours. Histoire et catalogue, Istanbul, Isis Press, 1985. 
l'unica rivista della comunità pubblicata continuativamente fino agli anni Settanta del Novecento, pur avendo rinunciato a una vocazione prevalentemente culturale.

In questo periodo merita invece qualche attenzione la tormentata vicenda del quotidiano «La Turquie», fondato dal giornalista italiano Guglielmo de Bondini nel 1906. Il progetto editoriale di de Bondini non ebbe un esito felice, la testata non si impose mai come organo ufficiale della cultura o degli interessi politici, finanziari e commerciali italiani, come il direttore-proprietario avrebbe voluto, nonostante un milieu politico e sociale almeno teoricamente disposto, anzi desideroso di aver finalmente un giornale italiano di ampio respiro, prezioso strumento di diffusione, oltre che di notizie, della lingua italiana. L'analisi di questo fallimento, tuttavia, può contribuire a fare un po' di luce su alcune dinamiche in atto fra il 1905 e il 1908 tanto a livello nazionale quanto, soprattutto, all'interno della comunità.

Nell'agosto del 1905 de Bondini scrisse al Console italiano a Costantinopoli, il Cav. Ciapelli, affinché intercedesse per ottenere l'iradè imperiale, ossia l'autorizzazione ottomana necessaria per dare avvio alla pubblicazione del quotidiano. Così si presentava: «il sottoscritto, Guglielmo di Bondini, italiano, residente in questa città da molti anni datosi al giornalismo, vorrebbe fondare qui in Costantinopoli un giornale quotidiano, politico, finanziario e di commercio, italiano nello spirito e che potrà un giorno essere redatto tutto interamente in italiano: ma che ora, per poterlo far conoscere, bisognerebbe redigere parte in italiano e parte in francese: italiano però intanto il titolo: "La Turchia"» 38 .

A partire da questa richiesta, l'avventura editoriale di de Bondini fu costantemente accompagnata, da parte delle autorità governative e di buona parte della colonia, da un atteggiamento altalenante fra il concreto appoggio e un'aperta avversione. Questa prima richiesta di de Bondini fu inviata dal Console Ciapelli all'allora ambasciatore a Costantinopoli, il Marchese Imperiali di Francavilla, il quale a sua volta la inoltrò al ministro degli esteri, Tommaso Tittoni. Quest'ultimo rispose molto sinteticamente all'ambasciatore che per la necessaria autorizzazione si sarebbe rimesso al suo giudizio, e riferendosi al sostentamento economico dell'iniziativa, aggiunse, «è però evidente che, se il giornale non può essere vitale, è meglio che non se ne inizi la pubblicazione»39.

La chiosa di Tittoni faceva presagire una non scontata disponibilità, da parte del governo, di intervenire con finanziamenti a sostegno del giornale. Di fatto, de Bondini iniziò presto a scrivere numerose lettere a Zaccagnini, all'ambasciatore Imperiali e,

${ }^{38}$ ASDMAE, Ambasciata d'Italia in Turchia (1829-1937), b. 101, f.5.

39 Ibidem. 
tramite il gentiluomo di corte il Marchese di Villamarina, scrisse perfino alla regina madre, chiedendole, in occasione dell'uscita del primo numero (ottobre 1906), «un segno che valga a infondermi forza e coraggio per sopportare i sacrifizii impostimi, superarne le difficoltà e tenere, così, alto il nome italiano»40, oltre che una meno esplicita richiesta di aiuti più materiali. Da Stupinigi uno dei Cavalieri di corte di sua maestà scrisse laconicamente a Imperiali di riferire a de Bondini che la regina avrebbe potuto al massimo - e solo nel caso in cui questo fosse ritenuto «meritevole di tale segno della Reale benevolenza» - sottoscrivere un paio di abbonamenti, e aggiungeva di riferirgli inoltre di desistere dall'inviare «carte e giornali» al Marchese di Villamarina, deceduto oramai da anni. Il tono distaccato di queste missive avrebbe sempre accompagnato l'iniziativa di de Bondini, tanto in patria quanto presso la stessa colonia di Istanbul, come lui stesso ebbe a lamentarsi presso l'ambasciatore Imperiali nell'agosto del 1907: «Da tutte le istituzioni italiane, dalle grandi case bancarie, negozianti ed altro, il giornale non ha avuto una sovvenzione qualsiasi, e per gli abbonamenti stessi carità di patria vuole che io non faccia un apprezzamento [...]»41.

La Dante fu direttamente coinvolta nella vicenda, al punto che gran parte della corrispondenza fra il comitato locale e Roma in questo periodo riguardò proprio il quotidiano. Mizzi, sensibile per esperienza di vita e professionale alle questioni editoriali, riconoscendo il nesso che avrebbe dovuto legare l'esistenza della società e la presenza di un quotidiano italiano, scrisse nel marzo del 1907 a Zaccagnini una lunga lettera nella quale attribuiva la responsabilità del fallimento del progetto debondiniano innanzitutto alla stessa presidenza generale della Dante ${ }^{42}$.

\section{Ibidem.}

${ }^{41}$ Ibidem.

${ }^{42} \mathrm{La}$ "Turchia" è molto maltrattata dalla Dante non solo ma anche dal Governo italiano. Sebbene questo sia precisamente lo identico stato di cose del quale fruisce il mio Levant Herald, pure non posso non capire né disapprovare i tristi lamenti del de Bondini [...]. In un momento in cui si sta facendo tutto per risvegliare il sentimento e la favella d'Italia in queste regioni, eccoti spuntare sotto i migliori auspici un "giornale italo-francese". Gli italiani naturalmente lo devono tenere caro ed il Marchese Imperiali non ha omesso una sola circostanza nella quale non lo raccomandò come opera nazionale italiana. Ora quale è l'accoglienza che è stata fatta dalla Dante e dal governo italiano? Assolutamente nulla. La Dante, né come società, né come comitato locale non ha ancora, per così dire, salutato l'arrivo di un giornale italiano. Ora, tu sai meglio di me che gli scopi della dante sono, spargere la lingua e la coltura italiana ovunque. Se il giornale di Bondini pro tanto non isparge gran fatto di coltura italiana, egli sparge certamente ed indubitabilmente la lingua italiana. Sotto questo aspetto dunque ha diritto alla simpatia ed all'incoraggiamento della Dante. Ora tu sai meglio di me che la dante come comitato locale non può fare gran cosa pel Bondini, perché non è ricca [...]. Ma la cosa è bene diversa colla Dante in Italia. La società madre vede gli sforzi di Bondini, e se il governo non ha fatto nulla per lui, mi sembra che la colpa tutta sia della Dante la quale ha voce in capitolo in Italia e non perderebbe nulla se si mettesse un poco in campagna a prò di lui. [...] Lo "Stanboul" riceve una splendida sovvenzione dal governo francese, perché la "Turchia" non dovrebbe riceverne una dagli italiani? il Levant Herald non ha un soldo dal governo inglese, ma anche lo stato inglese non ha 
Nel novembre del 1907 Tittoni si mosse in favore di De Bondini, comunicando a Imperiali - che aveva già fatto ottenere a «La Turchia» un sussidio annuo dalla Banca Imperiale Ottomana 43 - di attivarsi per fare avere fondi al giornale, suggerendo che «sarebbe opportuno che venissero officiati la Società Commerciale d'Oriente, il Delegato italiano al Debito Pubblico - che può fare molto - le Camere di commercio italiane di Costantinopoli, Salonicco, Smirne, i R.R. consolati d'Italia nell'Impero ottomano, e quante altre istituzioni italiane possano trarre, direttamente o indirettamente, vantaggio da una bene intesa pubblicità politica od economica», e ancora nel luglio del 1908, Tittoni, su sollecitazione di Imperiali, concesse un sussidio di cinquemila lire al giornale, «a carattere di concessione straordinaria, fatta in via di esperimento, e senza impegno per l'avvenire»44.

Il 1908 segnò la fine di questo esperimento, decretandone il sostanziale fallimento. Con l'annessione della Bosnia da parte dell'Austria-Ungheria e a seguito dei provvedimenti per la libertà di stampa introdotti con il ripristino della Costituzione dopo la rivoluzione dei Giovani turchi, de Bondini impresse al suo giornale un indirizzo politico marcatamente antitriplicista, che spinse anche l'ambasciatore Imperiali, che fin dall'inizio era stato principale interlocutore e sostenitore dell'iniziativa nell'ambiente governativo (attirando sull'ambasciata non poche critiche da parte della colonia), a ritenere oramai sconveniente qualsiasi sostegno al giornale - «Io avevo propugnato l'idea di un giornale italiano a Costantinopoli, in altri tempi e in altre condizioni, cioè quando, con la censura, si era al riparo di ogni pericolo di disquisizioni politiche di alcun genere». Posizione che il ministro Tittoni fece sua e chiaramente espresse allo stesso Imperiali il 23 novembre 1908: «La condotta del de Bondini ha sufficientemente dimostrato che non si possa prendere in seria considerazione l'offerta dei suoi servizi e

né viste né ambizioni in Turchia e se ne infischia dei giornali in generale e del Levant Herald in particolare. [...] E rifletti che se il governo non dà prova di buona volontà a l'interesse per il giornale italiano locale, non si può, non si deve pretendere che la colonia lo prenda a ben volere. Tu sai come vanno le cose qui. Se il movimento viene comunicato dall'alto, i nazionali, come le pecore del nostro Padre Dante, seguiteranno a capo fitto, e quello che la prima fa, le altre faranno. Io ti parlo in questo senso, come ho sempre parlato a tutti, sebbene che il mio interesse personale sia precisamente che voi seguitiate a trattare la Turchia come l'avete trattata fin d'ora. Anche io ho un giornale e, naturalmente, non ho nessun interesse a farne prosperare un altro. Ora tu sai benissimo quali siano i miei veri sentimenti in proposito. Tu sai che ho avuto un fratello che ha lasciato la sua preziosissima vita dopo una lotta omerica di 27 anni in difesa della lingua italiana e tu sai che egli mi ha lasciato cotesto retaggio. E lo eseguisco fedelmente, sinceramente e del mio meglio. [...] Fa dunque del meglio per Bondini. Egli mi ha pregato di scrivertene ed io te ne scrivo in tutta coscienza. ASDA, fasc. Costantinopoli 1895-1921, $167 \mathrm{~A}$.

43 ASDMAE, Ambasciata d'Italia in Turchia (1829-1937), b. 101, f.5.

44 Ibidem. 
sempre più mi convinco che sia meglio di non aver rapporti con lui. Rimane soltanto il vivo rincrescimento di avergli corrisposto una somma non indifferente» 45 .

La dichiarazione di Tittoni, che suonava come l'epigrafe funebre dei legami fra de Bondini e il governo del Regno, comportò in realtà solo un'interruzione di questi ultimi. Con l'avvicendamento di Antonino di San Giuliano a Tittoni al ministero degli esteri, nel 1910, e quello di Camillo Garroni a Imperiali all'ambasciata, «La Turchia» fu presentato dal nuovo ambasciatore come «organo ufficioso dell'Ambasciata, cui non ha mai rifiutato il proprio concorso »46, riuscendo così a ottenere dal ministero degli esteri una sovvenzione mensile di milleduecento lire. De Bondini sospese poi le pubblicazioni nel giugno del 1915 quando, con l'ingresso dell'Italia in guerra accanto all'Intesa, la censura ottomana non consentì più la pubblicazione di articoli che non fossero in favore degli Imperi centrali. È difficile stabilire se le cause del disinteresse da parte degli ambienti romani, governativi e della Dante, debba attribuirsi esclusivamente alla personalità di De Bondini, personaggio indubbiamente controverso e discusso all'interno della comunità italiana di Istanbul. Certamente, in linea teorica, il comitato di Costantinopoli avrebbe potuto godere di straordinarie attenzioni nella particolare fase che stava attraversando la Società a livello nazionale.

Bisognerà aspettare il 1920 perché la Dante si doti di un proprio organo di stampa. A partire dal maggio di quell'anno, infatti, fino al maggio del 1924, si dispone di una fonte preziosa e dettagliata per seguire le attività dell'associazione nella comunità: il «Bollettino della Dante. Organo del comitato di Costantinopoli». Annunciato da Joli a Zaccagnini a gennaio47, il primo numero si aprì con il programma del comitato, che ribadì come dovere di ogni italiano dimorante fuori dai confini della patria doveva essere usare, conservare e diffondere la lingua nonché «persuadere chiunque che la Dante Alighieri è inspirata unicamente dal più schietto sentimento d'italianità e che è aliena da qualsiasi competizione politica e religiosa»48. Il Bollettino conteneva inoltre un articolo sull'attività della Dante in Italia e all'estero dalla sua fondazione, l'elenco dei 63 soci perpetui - ossia quanti versavano una soluzione unica di 150 Lire italiane -

\footnotetext{
45 Ibidem.

46 Ibidem.

47 «Un solo foglietto di 4 pagine, col quale si farà propaganda dando conto ai soci di ciò che fa il consiglio, di ciò che fanno, in ristretto succinto, gli altri comitati, notizie sui corsi, sulla biblioteca, libri ricevuti, donazioni, movimento soci, brevi recensioni di pubblicazioni letterarie e di conferenze [...]. Io mi riprometto un risultato eccellente di propaganda nella Colonia nostra e nelle altre di qui con questa pubblicazione - modesta da principio - ma che può avere grande sviluppo, tanto più che, salvo quel povero bollettino della camera di commercio, fatica particolare di Melia, qui non si stampa una parola d'italiano», Joli a Zaccagnini, 11 gennaio 1920, ASDA, fasc. Costantinopoli 1895-1921, 167A.
}

48 Bollettino, I, 1/1920, p. 1. 
l'annuncio della ripresa dei due corsi femminili, notizie sulla biblioteca circolante e un articolo in cui si riportava la cronaca della festa annuale commemorativa della fondazione della Dante. Infine, le ultime pagine erano dedicate agli sponsor, l'uso sapiente dei quali, come scrive lo stesso Joli, consentì di coprire tutte le spese per la pubblicazione. Quel che appare subito evidente scorrendo l'elenco dei nomi o dei luoghi che riempiono le pagine pubblicitarie di questo primo numero del Bollettino, è la valenza anche politica che essi esprimono, che va oltre la stretta funzione di finanziamento e che a ben guardare stride con le dichiarazioni di apoliticità del programma del comitato - le stesse, tra l'altro, della Società a livello centrale.

Concepito come strumento di penetrazione culturale, il Bollettino fu infatti sostenuto dai principali protagonisti della parallela azione di espansione economica e commerciale italiana, vale a dire la Banca italiana di sconto, la Banca commerciale italiana, il Banco di Roma, la Riunione Adriatica di Sicurtà, la Società italiana di servizi marittimi, l'Istituto Nazionale delle Assicurazioni, la società di trasporti e comunicazioni Intercontinentale, le Assicurazioni Generali di Venezia e il Lloyd Triestino, tutti organismi che ricoprirono un'incisiva funzione politica nei territori di interesse coloniale o inclusi nel raggio d'azione della spinta imperialistica. Del resto, la pretesa di mantenere una posizione apolitica venne ribadita proprio nel 1920 anche a livello nazionale, dove da tempo era in corso uno scontro fra due correnti interne, una a favore dell'intervento attivo della Dante anche nel territorio nazionale - e non solo nell'ambito dei comitati esteri, come sanzionato dallo Statuto - l'altra invece aperta a una partecipazione attiva dei comitati nella vita nazionale in occasioni di significativi avvenimenti anche politici. Questa seconda linea, respinta fino al 1920, prevalse inevitabilmente dopo la guerra, ancora accompagnata da una condizione di apoliticità destinata però a infrangersi presto contro le ingerenze (e le seduzioni) dell'ascesa fascista ${ }^{49}$ : una seduzione che nel comitato di Istanbul avrebbe dato presto i suoi frutti.

Per quanto concerne le scuole italiane a Istanbul volute dalla Dante, la loro vicenda seguì l'andamento del dibattito nazionale. Dopo il 1903 il tentativo di imprimere definitivamente alla Dante un'impostazione basata sugli ideali democratici risorgimentali lasciò il posto a una visione che, da questo momento in poi, avrebbe risposto prevalentemente alle tensioni imperialistiche50. Neppure la crescente attenzione per i grandi centri d'emigrazione transoceanica riuscì a far prevalere le ragioni sociali dell'azione della Dante sul vigore della spinta espansionista. Nel XV congresso nazionale fu esplicitamente dichiarato che le scuole del Levante non avevano

49 PISA, Beatrice, op. cit., p. 356.

50 PISA, Beatrice, op. cit., p. 14. 
in realtà alcuna utilità in quei paesi in cui l'emigrazione propriamente detta non era più numericamente interessante, perché gli italiani presenti erano oramai prevalentemente figli di quanti, legati al mondo del commercio, si erano stabiliti in quei territori51, ma già nel congresso nazionale dell'anno successivo, il Levante, e più in generale il mondo musulmano si imposero nuovamente all'attenzione dell'assemblea. Le relazioni di Enrico Insabato ${ }^{52}$ e i suoi progetti per l'insegnamento nei paesi musulmani e quella sulle scuole italiane del Levante di Giuseppe Solimbergo riportavano entrambe l'attenzione sui potenziali vantaggi che un sistema di scuole italiane nel Levante avrebbe potuto restituire in termini politici ed economici. $\mathrm{E}$ a quanti obiettavano che $\mathrm{i}$ numeri dell'emigrazione imponevano maggiori interventi nelle Americhe, Solimbergo replicava che la valenza politica dell'area mediterranea costituiva un fattore imprescindibile:

le altre nazioni hanno altro: dei continenti interi popolati della loro razza, vasti imperi coloniali, colossali interessi industriali e commerciali noti al mondo. Noi non abbiamo in vista che il Mediterraneo - come in antico. Quello è il nostro naturale campo, e il più importante, di osservazione e di operazione: con l'Italia che vi si slancia attraverso; con tante vive traccie [sic] che vi abbiamo lasciato dappertutto, nel nostro passato, sulle isole e sulla terraferma - rovine che ora accennano come a rianimarsi, nel sentimento e nella fede dei nostri, che vi stanno intorno, con la coscienza di una patria che va crescendo nella sua produzione e nei mezzi della sua difesa, che comincia a contare; con la penisola Balcanica che rasenta l'Adriatico; con l'Africa che si arriva a vedere53.

Più analitica e più orientata verso il delicato problema della comprensione dell'Islam - tematica che si sarebbe posta in termini più concreti dopo la guerra di Libia ma già presente nel dibattito politico italiano a partire dall'ultimo ventennio dell'Ottocento 54 - si presentava la relazione di Insabato. Sostenitore di un modello di scuola italiana all'estero laica, specie nelle province musulmane, Insabato proponeva di creare scuole differenziate per italiani e per indigeni e intanto di introdurre dei corsi di

${ }^{51}$ SALVETTI, Patrizia, op. cit., p. 90.

${ }^{2}$ Stretto collaboratore di Giovanni Giolitti negli anni in cui fu ministro degli interni, operò principalmente in Egitto. Sulla figura di Insabato e sulla sua politica in questi anni cfr. IANARI, Vittorio, La politica islamica dell'Italia durante la Triplice Alleanza. L'attività di Enrico Insabato, in TRINCHESE, Stefano (a cura di) Mare nostrum. Percezione ottomana e mito mediterraneo all'alba del '9oo, Milano, Guerini, 2005, pp. 199-246.

53 SOLIMBERGO, Giuseppe, Le scuole in Levante. Relazione al XVI Congresso della "Dante Alighieri”, Roma, Tipografia La Sapienza, 1905, p. 11.

54 Cfr. MARONGIU BUONAIUTI, Cesare, Politica e religioni nel colonialismo italiano (18821941), Milano, Giuffrè, 1982. 
giurisprudenza m, usulmana al fine di incoraggiare i locali ad inviare i propri figli in scuole percepite come amiche e rispettose della religione del luogo55.

Considerando la virata nazionalista e l'attenzione verso il mondo mediterraneo, ci si aspetterebbe in questo periodo un'intensa e organizzata attività del comitato di Costantinopoli, invece se si mettono a confronto le linee programmatiche emergenti dagli atti dei congressi nazionali e la corrispondenza inviata dal comitato di Istanbul a palazzo Firenze, si riscontra come alle strategie di diffusione della lingua e di propaganda nazionale elaborate presso il comitato centrale corrispose in realtà un'attività a Istanbul in cui l'elemento prevalente fu la forte conflittualità interna fra le personalità più in vista della colonia. Dopo la fine della presidenza Mizzi e una breve parentesi di D’Agostino, fu eletto il marchese Alberto Theodoli di Sambuci, fondatore della locale sede del Banco di Roma e futuro senatore del Regno, partito però poco dopo l'elezione, lasciando il suo posto all'architetto Edoardo De Nari, il quale impresse effettivamente un indirizzo più decisionista alla Dante dopo un periodo di tendenziale immobilità, ma fu a sua volta al centro di numerose polemiche sollevate soprattutto rispetto ai suoi atteggiamenti esterofili.

Alla vigilia della rivoluzione dei giovani turchi quindi, la Società aveva all'attivo 283 soci, finanziava corsi per signore e signorine, aveva sospeso i corsi di italiano nelle scuole straniere - «un'esperienza di alcuni anni che pare non abbia dato risultati soddisfacenti» ${ }^{6}$ - promuovendo però corsi diurni destinati a studenti di istituti stranieri. Questi ultimi furono frequentati da novantuno allievi, e a essi si aggiunsero corsi serali destinati a impiegati e artigiani cui si iscrissero in ventiquattro. I centoquindici frequentanti queste due tipologie di corso erano tutti stranieri, e proprio sulla maggiore partecipazione dei non nazionali alle attività della Dante, De Nari, recuperando l’impostazione che era già stata di Zaccagnini, puntò per un rilancio delle attività della Società ${ }^{57}$. Quanto alle iniziative in ambito scolastico, allo stesso Zaccagnini De Nari scrisse rilanciando l'idea, più volte carezzata nella comunità, di fondare una scuola elementare gratuita:

\footnotetext{
55 SALVETTI, Patrizia, op. cit., p.98.

${ }^{56}$ Come scrisse Theodoli a Zaccagnini, ASDA, fasc. Costantinopoli 1895-1921, 167A.

57 «Ragiono e dico che i 200 soci della Dante degli anni passati erano in gran parte italiani, e più di tanti non ne avremmo mai avuti, che se volevamo aumentare sensibilmente il numero bisognava cercare fra gli stranieri ed invogliarli. Gli stranieri si faranno soci della Dante per ciò che la Dante darà loro e pagheranno pure per la festa a pagamento in riconoscenza del profitto avuto, ma è illogico pensare che stranieri si faccian soci per gli scopi patriottici della Dante, o per istruirsi in conferenze e commedie o in letture nelle quali poco ci capiscono. Alle prime 2 feste furono ogni sera 300 gli intervenuti. Su 300 le assicuro che ce n'erano appena 100 che capivano l'italiano». ASDA, fasc. Costantinopoli 1895-1921, 167A.
} 
Ultimo e più importante dei progetti. Creazione di una scuola italiana, popolare gratuita a Galata. E punto! [...] Dove, secondo lei, la Dante dovrebbe manifestare il più d'attività? Per me in Oriente - Egitto - Costantinopoli - Albania. Ora in Turchia, a Costantinopoli c'è la doppia utilità, influenza morale e beneficio materiale. Colla conoscenza della lingua c'è la conoscenza del paese e con questa quella dei suoi prodotti, delle sue potenzialità artistiche, industriali, commerciali. [...] Tutti grideranno che i miei progetti son pazzi (come chi li fa) ma io li sosterrò, se lei mi dà buone speranze, e se questo consiglio non ha l'energia voluta, l'anno venturo farò scegliere altri elementi o più forti che mi secondino, o più deboli che mi lascino fare ${ }^{5}$.

La scuola elementare fu probabilmente, in questa prima fase, una delle idee meglio concepite dal Comitato. Nel 1909 il ministero degli esteri avallò la proposta della sua istituzione, la già esistente scuola elementare di Pera divenne a pagamento e al comitato, sprovvisto dei mezzi economici necessari per creare dal nulla l'edificio scolastico, fu messo a disposizione gratuitamente il terreno adiacente al regio ospedale italiano e la garanzia di un contributo dal ministero. Progettata da Mongeri e De Nari e costruita in tempi sorprendentemente brevi, la scuola fu aperta il 10 dicembre 1910.

L'11 marzo 1909 Emilio Marchione, direttore della nuova scuola elementare della Dante, inviava al dottor Riccardo Zeri, allora presidente del comitato locale, la prima, lusinghiera relazione sull'andamento dell'istituto:

Gl'insegnanti comandati a prestar servizio in essa furono due, ma per il numero sempre crescente di allievi, presto si rese indispensabile l'opera di un nuovo educatore, che fu fatto venire dall'Italia, non trovandosi in città persona idonea al delicato ufficio. Oggi la nuova scuola conta 224 alunni, ed a misura che se ne conosce l'indirizzo educativo, le decenza del locale e la bontà della refezione, aumentano le iscrizioni; cosicché comincia persino ad imporsi la necessità di provvedere alla nomina di un altro insegnante59.

Marchione riferiva anche la ripartizione secondo la nazionalità dei 244 iscritti, 96 italiani e 123 ottomani, di questi ultimi poi, 60 erano turchi, 12 armeni, 25 israeliti e 26 greci. A questi si aggiungevano 2 russi, 1 austriaco, 1 francese e un persiano. Si istituirono quattro classi che seguirono gli stessi programmi delle scuole del Regno, «con qualche lieve modificazione per renderli più conformi alle esigenze ed ai bisogni locali». Dalle 14 alle 16, «dopo due ore di riposo assegnate alla refezione e alla

$5^{8}$ De Nari a Zaccagnini, 8 dicembre 1907, ASDA, fasc. Costantinopoli 1895-1921, 167A.

59 Emilio Marchione a Riccardo Zeri, 3 marzo 1911, ASDA, fasc. Costantinopoli 1895-1921, 167A. 
ricreazione, vengono svolti i programmi delle materie complementari, destinate ad imprimere il carattere particolare della scuola, e cioè: il turco, il francese, il lavoro manuale, il disegno industriale, la plastica e la musica», queste ultime tre materie, specificava Marchione, erano insegnate a titolo gratuito. La scuola dunque rispose fin dalla sua istituzione alla missione di mantenere l'italianità fra i connazionali e diffondere lingua e cultura italiana fra le popolazioni autoctone e straniere, eppure già dal maggio del 1911 Marchione scriveva a Zaccagnini che le pesantissime difficoltà economiche avrebbero presto minato l'esistenza della scuola se il governo italiano non fosse intervenuto con sussidi congrui, non solo, ma riferiva di un atteggiamento inizialmente poco propenso da parte delle autorità consolari per la concorrenza che la scuola della Dante stava esercitando nei confronti della regia scuola elementare, atteggiamento divenuto favorevole, scrive ancora Marchione, dopo che il Console ebbe verificato l'efficienza dell'istituto ${ }^{60}$. L'intervento del Console presso il ministero degli esteri non diede frutti, come scrive il presidente Zeri nella relazione di luglio sulle attività svolte dalla Società durante l'anno, relazione che, dopo avere illustrato i risultati eccellenti della scuola, si concludeva con una sorta di ultimatum al governo, il cui mancato intervento avrebbe comportato inevitabilmente la chiusura della scuola ${ }^{61}$.

L’ipotesi di dover porre fine a un'iniziativa che stava dando ottimi frutti, perfettamente rispondente alle oramai decennali aspirazioni espansionistiche del Paese, sorta inoltre in una delle zone di maggiore interesse politico e commerciale e in un periodo di fortissima rinascita del sentimento nazionalista, sia a livello governativo sia presso la direzione centrale della Società, si presentava quanto meno come un'anomalia, la cui origine si può probabilmente rintracciare in una sorta di profonda incomprensione fra la base dei comitati locali e il governo centrale ${ }^{62}$. La chiusura della

6o Marchione a Zaccagnini, 16 maggio 1911, ASDA, fasc. Costantinopoli 1895-1921, 167A.

61 «Il risultato della scuola popolare è stato veramente superiore ad ogni previsione. Sorta per iniziativa di pochi, in mezzo alla diffidenza generale della colonia italiana che credeva non essere il quartiere adatto per una scuola, l'affluire immediato degli allievi sia italiani sia di altre nazionalità, venne subito a confermare le giuste previsioni dei fondatori. Non solo osservammo il numero degli allievi, ma ci colpì il progresso in breve tempo dei loro studi [...]. Voi sapete il risultato finale degli iscritti nella scuola, questi sono stati 275, di cui ben 206 assidui e di questi 99 erano italiani, 126 ottomani, 44 greci, e 2 persiani; divisi per religione si contarono: 91 cattolici, 90 musulmani, 52 israeliti, 42 ortodossi. Gli esami finali ci hanno infine data la prova del progresso reale fatto dagli alunni negli studi impartiti [...]. Ottimi risultati, ottimi insegnanti che ci lascerebbero fare le migliori previsioni per l'avvenire, se il punto nero non venisse dal nostro bilancio. [...] In tali condizioni, visto l'indifferenza del R. Ministero a noi non resterebbe che chiudere la scuola. Ci conforta il pensiero che avendo sacrificato tutto il suo capitale nell'impianto della scuola popolare, la Dante ha potuto far sorgere in pochi mesi una scuola così prospera e popolata come mai la scuola elementare governativa è stata in più di 20 anni». ASDA, fasc. Costantinopoli 1895-1921, 167A.

62 Questo tipo di difficoltà è riferito, per esempio, da Marchione, il quale riferendo di incomprensioni con il Console, riferiva all'Ambasciatore: «Ho creduto necessario d'esporre 
scuola sopravvenne comunque nel 1911, imposta infine non dalla mancanza di fondi ma dalla deflagrazione della guerra di Libia, per la quale la colonia italiana si trovò improvvisamente a vivere in territorio nemico. È in questa occasione che per la prima volta, nella documentazione a diposizione fanno irruzione elementi di vissuto relativi ai rapporti della colonia con l'ambiente esterno, le corrispondenze del presidente Zeri a Zaccagnini offrono uno spaccato di quotidianità durante i mesi di conflitto che, pur immerso in un clima ovviamente teso, non rivela momenti particolarmente drammatici ${ }^{63}$ :

[...] il punto nero era però la questione finanziaria, che, dopo lunghe trattative col R. Ministero e col comitato centrale, si era potuta infine favorevolmente risolvere per l'anno scolastico 1911-1912. Ma sopravvenne la guerra. Ci trovammo allora costretti a chiudere la scuola; il Ministero e il comitato centrale ci sospesero il sussidio, mentre il nostro bilancio era già in deficit, e mentre avevamo presi degli impegni con alcuni insegnanti. Dopo lunghe pratiche col Comitato centrale, questi indennizzò l'insegnante D'Ardes che noi avevamo già impegnato per l'anno scolastico e indennizzò l'insegnante di francese. Non fu però indennizzato l'insegnante di turco, malgrado che questi, avendo presi impegni con noi, per la nostra scuola, avesse rinunziato ad altre lezioni, e, essendo straniero, più degli altri avrebbe meritato qualche riguardo. [...] Ora ci domandiamo; la scuola popolare deve continuare o no? Il consiglio è stato sfavorevole alla riapertura della scuola, primo perché l’impresa è grande in paragone delle risorse finanziarie della società, secondo non crede che il momento sia opportuno per avere due scuole; tutti i nostri

quanto sopra, non per risentimento o rancore, né per dimostrare come viene gratificata alle volte l'opera concordemente apprezzata e lodata di un insegnante, ma solo per dimostrare la necessità d'un accordo fra la direzione generale delle RR. Scuole all'estero e il comitato centrale della Dante Alighieri, perché, se non bastano le tassative disposizioni del regolamento, con opportune istruzioni, si compiacciano di stabilire i limiti delle competenze e delle ingerenze del R. consolato generale sulla scuola popolare di Costantinopoli, a fine di evitare in seguito nuovi equivoci e malintesi, i quali potrebbero danneggiare una scuola che, sorta sotto lieti auspici e circondata da molte simpatie, sembra destinata ad un ottimo avvenire», ASDA, fasc. Costantinopoli 1895-1921, 167A.

63 «Caro Zaccagnini, da quattro giorni qui non abbiamo che notizie disastrose per l'Italia; Tripoli ripresa dai turchi, Bengasi riespugnata e così via. Sono immolati montoni per ringraziare Allah della vittoria e con che aria [parola incomprensibile] ora siamo guardati se lo può ben immaginare lei [...]. Notizie di qui non gliene do': finora siamo restati tranquilli e non ci hanno ancora trucidati, né evirati [...]. Solamente mi volevano chiudere l'ospedale e poi non l'han fatto, e nel quartiere turco dove si trova l'ospedale sono furibondi. Non posso andare da casa mia all'ambulatorio perché quei bravi turchi che prendono il caffè in quelle bottegucce hanno voglia di bastonare gl'italiani. Io pensando che è meglio di non dar loro tale fatica passo da un'altra strada. Ed ora le chieggo aiuto per la scuola popolare che non è aperta. Io della Dante non ho più un soldo; la cassa è vuota e non è questo il momento di domandar le quote ai soci. Ma il bidello lo devo pagare, l'abbonamento dell'acqua anche; poi ci sono delle altre piccole spese [...], per ora pago io, ma se la guerra continua dovrei licenziare il bidello, e mentre tutti i lavoranti italiani soffrono la fame non vorrei farne soffrire uno in più [...]». Zeri a Zaccagnini, 2 novembre 1911, ASDA, fasc. Costantinopoli 1895-1921, 167A. 
sforzi devono ora farsi, onde la scuola governativa risorga rigogliosa per qualità e per numero d'alunni; quando ciò sarà avvenuto si cercherà di risolvere i problemi della scuola a pagamento e della scuola popolare» 64 .

In effetti la soppressione della scuola della Dante risanò la regia scuola elementare. Secondo le cifre riportate dall'Annuario scolastico, nel 1911 risultavano iscritti alla scuola elementare governativa 23 alunni, mentre alla scuola popolare della Dante 245. Soppressa quest'ultima e riprese le attività scolastiche dopo la conclusione del conflitto, nel 1913 gli iscritti alla scuola regia furono 144, tornando sostanzialmente agli stessi numeri fatti registrare prima dell'apertura della scuola della Dante ${ }^{65}$. Purtroppo non si dispone della documentazione dettagliata delle iscrizioni per entrambe le scuole, ma si può verosimilmente dedurre, visto l'andamento dei numeri, che si trattò degli stessi iscritti che passarono da una scuola all'altra, ed essendo i programmi di studio, in entrambi i casi, ricalcati su quelli governativi, la brevissima parentesi della scuola elementare popolare della Dante non rappresentò tutto sommato un elemento di discontinuità nell'ambito del progetto educativo italiano ad Istanbul. Fu però un'esperienza interessante rispetto agli equilibri interni della parte più attiva della comunità italiana. Contrariamente a quanto stava accadendo in quegli stessi anni intorno alla vicenda de «La Turchia», che aveva evidenziato ed esacerbato alcune tensioni fra i soci - tensioni che, si vedrà successivamente, negli anni della fascistizzazione della Dante sarebbero riemerse - la gestione della scuola italiana fu caratterizzata da una laboriosa e unanime collaborazione. La scuola, non più operativa dal 1912, fu chiusa definitivamente nel 1913 e lo stabile venduto al governo.

Del resto la situazione nel Paese era radicalmente cambiata. Mentre il nazionalismo italiano cercava le proprie declinazioni più consone ai suoi obiettivi nell'Impero, il nazionalismo turco aveva fatto irruzione nell'età hamidiana fra il luglio del $1908 \mathrm{e}$ l'aprile del 1909. La rivoluzione dei Giovani turchi aveva imposto prima il ripristino della costituzione del 1876 e poi l'abdicazione di Abdulhamid. Quali furono le ripercussioni di questi cambiamenti nella vita della Dante di Istanbul? A giudicare dalla corrispondenza conservata, si direbbe nessuna. Fatta eccezione per un appunto inviato a Roma da Luigi Joli, capo contabile alla manifattura dei tabacchi, in cui riferiva che «le sorti della Regìa sono indecise, poiché i Giovani turchi, malgrado le molte belle cose

64 Relazione sull'andamento del comitato di Costantinopoli negli anni 1911-12, ASDA, fasc. Costantinopoli 1895-1921, 167A.

65A partire dall'anno scolastico 1902-1903 fino al 1909 gli iscritti alla scuola elementare governativa furono rispettivamente $265,219,156,157,156,124$ Cfr. le corrispondenti pubblicazioni dell'Annuario delle scuole italiane all'estero governative e sussidiate, Ministero degli affari esteri, Roma. 
fatte, sono poco propensi a rinnovare la concessione nel monopolio tabacchi» ${ }^{66}$. Per quanto ci è dato sapere, il vento rivoluzionario del luglio 1908 passò dunque sulla Dante senza stravolgere la vita del sodalizio, le cui attività non subirono variazioni di programma. Risonanza assai maggiore ebbe presso il comitato, anche per la provenienza di molti dei suoi soci, la notizia del terremoto che devastò Messina e Reggio Calabria nel dicembre dello stesso anno, in seguito alla quale tutte le attività della Dante vennero sospese escluse quelle relative alle scuole.

\section{Le donne, i giovani, "Italica Gens". Prove di nazionalizzazione, 1912-1922}

La condizione economica del nostro comitato è peggiore di quando ebbi l'onore di scriverle lo scorso novembre. Causa la guerra balcanica il paese è nella più grande miseria, ed è difficile di incassare le quote, e tanto più di dare una festa in favore della Dante. [...] Il corso d'italiano alle signore e signorine è cominciato sin dal gennaio u.s.; l'affluenza delle allieve della migliore società di Pera è stato numerosissimo. Il primo corso è frequentato da 50 allieve, e il corso superiore da 30. Ł̀ stato anche organizzato un ciclo di conferenze e tra breve ne sarà data una anche dal nostro console generale cav. Tritoni ${ }^{67}$.

Così il dottor Riccardo Zeri riassumeva la situazione della colonia e del comitato alla presidenza generale nel marzo 1913. Tutta la prima parte del decennio 1912-1922 fu dominata dal clima bellico che si estese dalla guerra di Libia al primo conflitto mondiale senza soluzione di continuità, e dal giugno del 1915 al luglio del 1919 non si conserva alcuna comunicazione fra Istanbul e la sede centrale. Fino al 1915 tuttavia, le attività della Dante, pure dovendo svolgersi fra difficoltà ancora più accentuate rispetto agli anni precedenti, specie dal punto di vista economico, procedettero, e in alcuni casi anche con successo.

I corsi femminili nel febbraio 1914 fecero registrare un aumento tale che fu necessario creare quattro classi distinte e affidarle ad altrettanti insegnanti. ì interessante che fin dalla sua istituzione, il corso di lingua destinato alle donne ebbe sempre un numero elevato di iscritte, tanto che questi corsi furono gli unici a non essere mai sospesi - al di là dei periodi di guerra e di conseguente cessazione totale delle attività. Fino ai primi anni Venti non si dispone di informazioni più dettagliate

66 Joli a Zaccagnini, ASDA, fasc. Costantinopoli 1895-1921, 167A.

${ }^{67}$ Riccardo Zeri alla presidenza centrale, 3 marzo 1913, ASDA, fasc. Costantinopoli 1895-1921, $167 \mathrm{~A}$. 
relativamente alle iscritte, non è dato dunque sapere di che nazionalità fossero, di che estrazione sociale e cosa le spingesse ad imparare la lingua italiana. Successivamente, si riferirà che le partecipanti furono prevalentemente donne di nazionalità europea, soprattutto greche, ma non turche, a parte qualche rara eccezione. Certamente a livello propositivo la partecipazione attiva dell'elemento femminile della comunità nel comitato di Istanbul non sembra essere stata particolarmente significativa. In un appunto del 1901, probabilmente di Mizzi, si annotavano le imminenti proposte del comitato e fra queste si annunciava che sarebbe stato costituito un "comitato di signore», proprio «allo scopo di porgere alimento nuovo e perenne ai corsi d'italiano già da qualche anno iniziati da questo comitato», oltre che per «promuovere e bandire concerti, rappresentazioni, conferenze a pagamento, ricreatori popolari, allo scopo infine di compiere qualunque atto che il consiglio, l'assemblea, le circostanze e il discernimento innato degli animi femminili suggeriranno» ${ }^{68}$. Qualche settimana dopo, in effetti, fu inviata una comunicazione firmata da sette donne, molte delle quali erano mogli o familiari dei dirigenti del comitato, con la quale si sollecitavano tutte le signore italiane residenti a Istanbul a dare vita al comitato femminile. A questo fine erano invitate a un'assemblea generale dove «verranno esposte le ragioni e gl'intendimenti per cui la donna è chiamata a partecipare ai lavori di una istituzione così nobile ed elevata ${ }^{69}$. Oltre questo invito, nella documentazione non c'è più traccia del comitato. La Dante di Istanbul d'altronde, pur non mancando di dedicare attenzioni al modo femminile $7^{70}$, non si distinse mai per posizioni particolarmente progressiste. Tale era stata del resto a inizio secolo la posizione della Società centrale, che promosse sì proprio in questi anni un sempre maggiore coinvolgimento della componente femminile, ma solo in quanto questa era ritenuta ancestralmente in grado di risultare efficiente nell'opera di propaganda ${ }^{71}$. Si dovrà aspettare la svolta nazionalista, dopo il primo decennio del Novecento, per una ridefinizione del ruolo femminile oramai strettamente connesso con la missione di sostegno alla patria, e definitivamente consacrato, dopo la grande guerra, alla visione epica del sacrificio della madre ${ }^{72}$.

La biblioteca della Dante del resto, assorbita successivamente da quella della Società operaia e ancora consultabile nella sede di quest'ultima, non offriva alle socie

\footnotetext{
68 Proposte del comitato, Appunto, 26 marzo 1901, ASDA, fasc. Costantinopoli 1895-1921, 167A. 69 ASDA, fasc. Costantinopoli 1895-1921, 167A.

70 Nel 1897 l'avvocato Rosasco conferì su "La dignità della donna" e nel 1898 Carmelo Melia su "La donna nella società", ASDA, fasc. Costantinopoli 1895-1921, 167A.

${ }^{71}$ Cfr. PISA, Beatrice, op. cit., pp. 162 e sg.

${ }^{72}$ Ibidem, pp. 280 et seq.; MOSSE, George L., Sessualità e nazionalismo. Mentalità borghese e rispettabilità, Roma-Bari, Laterza, 1996, p. 128.
} 
letture di ampio respiro. Nel primo numero della rivista «Italia!»73, per esempio, la rubrica La pagina della donna italiana ospitò un articolo di Teresita Guazzaroni significativamente intitolato Dall'ufficio alla casa, L'esperienza di una donna, in cui l'autrice fa confessare alla sua amica la sua scelta di tornare, dopo una parentesi lavorativa come segretaria in un ufficio - non a caso in Inghilterra, terra delle suffragette, dove la protagonista del racconto si era laureata e aveva infine trovato lavoro - alle faccende casalinghe. Concludeva la Guazzaroni: «La breve storia della giovane donna che dalle cure dell'ufficio, attraverso piccole tristi esperienze torna al lavoro sempre fresco e nuovo della casa è una storia vera. Io non faccio neppure i commenti»74. Questa e altre letture non dissimili proponevano un modello di donna remissiva, posta a distanze siderali dalle coeve battaglie di Emmeline Pankhurst e assoggettata al giudizio strisciante di una pubblica opinione tutta al maschile che si limitava a esprimere una visione conservatrice nel caso delle italiane, ma si manifestava e si accaniva anche con veemenza sulla componente levantina della comunità femminile. La donna levantina di Istanbul era considerata irrimediabilmente corrotta dalla componente orientale, lasciva per sua natura, canonizzata in certa pubblicistica ed espressa negli umori popolari attraverso una serie di stereotipizzazioni entrate a far parte del comune linguaggio - «chi vuol far la sua rovina, sposi donna levantina»75. Immersa in questo clima, dai documenti a disposizione emerge come l'opera della parte femminile della colonia si esaurì, di fatto, nell'impegno in beneficenza, in attività assistenziali o di cura dell'infanzia, per esempio presso i refettori delle scuole elementari.

73 Il periodico fu fondato nel 1912 come integrazione al Bollettino generale della Dante, ritenuto troppo asettico ed eccessivamente incentrato sulle vicende interne della Società. PISA, Beatrice, op. cit., p. 165.

74 «Ero lieta di non dover rendere conto del mio tempo di cui mi pareva di essere assoluta padrona, mentre pur mi assoggettavo alle esigenze di un orario. Ma tale importanza davo al mio lavoro, che le ore dedicate ad esso mi parevano così preziosamente riempite, e così feconde che non osavo neppure a me stessa confessare la mia stanchezza. E con un profondo senso di compatimento io consideravo la sorte delle altre donne, fanciulle o spose, le quali erano costrette alla vita incolore imposta dalle esigenze domestiche. Ma poco a poco il mio entusiasmo si attenuò senza che neppure io riuscissi a rendermene conto. A un certo punto mi accorsi di sentirmi come straniera nella casa dove tornavo la sera, ove tutto era disposto senza il mio intervento che non poteva e non doveva esser necessario mai. Non avevo più tempo neppure per i miei libri di sociologia e di storia; e qualche volta, quando riuscivo ad aprirli, essi parevano parlarmi di un mondo lontano, che non avrei raggiunto mai, poiché quello in cui vivevo, che pure era il più ampio che potesse venir consentito alla mia attività femminea, non mi riconduceva, o per lo meno non mi richiedeva quasi mai quel che i miei libri mi avevano insegnato», «Italia!», I, 1912, p. 487.

75 CORSI, Carlo, op. cit., p. 62. «La generazione dei Levantini è come un anello intermedio che unisce l'Occidente all'Oriente: fra l'Europa e l'Asia v'ha un terreno neutro che è stato preso da loro: là si vengono ad incontrare due correnti opposte di civiltà e si corrompono scambievolmente. Così tante volte all'imboccatura dei fiumi, l'acqua dolce mescolandosi all'acque salse si guasta e riempie l'aria di pestifere esalazioni». Ibidem, p.67. 
Alla fragile esperienza del comitato femminile, si affiancò quella breve ma inizialmente più consistente di un sottocomitato studentesco, fondato nel novembre del 1913. Prima esperienza di questo genere fra tutti i comitati esteri della Dante, il sottocomitato era sorto per iniziativa del professor Giovanni Ferretti, insegnante dell'istituto tecnico commerciale e patrocinato anche dalla Società operaia. Come recitava il regolamento, esso era aperto a «tutti i giovani italiani o stranieri che frequentino scuole italiane o straniere a Costantinopoli e accettino lo statuto sociale e il presente regolamento. Tutti i soci a qualunque nazionalità appartengano dovranno parlare la lingua italiana e aderire consapevolmente agli scopi della Dante» ${ }^{6}$. Nella relazione che Mario Zelempopulo, presidente del sottocomitato inviava il 26 giugno a Roma, egli riferisce di 80 soci in regola con i pagamenti, di questi, 25 erano studenti provenienti dalla regia scuola tecnico-commerciale, 19 dal corso preparatorio per questo stesso istituto, 16 dalla regia scuola elementare maschile, 3 dalla regia scuola femminile italiana, 2 dal convito italiano dei salesiani, gli altri 15 da scuole straniere.

Questa prima esperienza di associazionismo giovanile in ambito scolastico nasceva di poco in ritardo rispetto alle prime iniziative che, entro i confini del Regno, stavano coinvolgendo a tutti gli effetti gli ambienti della gioventù italiana nel grande processo di costruzione identitaria nazionale ${ }^{77}$. Così come in patria la nazionalizzazione dell'infanzia andava sempre più imponendosi come «un fattore decisivo della nazionalizzazione delle masse» 78 , a Istanbul il tentativo di coinvolgere la fascia più giovane della colonia riproponeva in proporzione la stessa dinamica: nazionalizzare $\mathrm{i}$ giovani studenti per rafforzare il sentimento di appartenenza della colonia. E nuovamente, anche rispetto allo sviluppo dell'associazionismo giovanile ritorna in questo periodo il ruolo centrale dell'imperialismo e le conseguenti potenzialità insite nella comunità di Istanbul a questo riguardo. Ricorda ancora Gibelli che «l'imperialismo come fenomeno sociale e culturale complesso sconta una dipendenza dal controllo e dalla mobilitazione dell'immaginario. Le missioni imperiali devono essere raccontate con immagini fascinose per ottenere il consenso e suscitare le energie necessarie al loro sviluppo», e come in questo senso la guerra di Libia fu il primo grande evento catalizzatore prima della grande guerra. L'avvento della prima guerra mondiale interruppe presto l'azione del sottocomitato, che pure ebbe modo di intraprendere e portare a termine alcune iniziative, fra le quali la diffusione di riviste

${ }^{76}$ Regolamento interno del sottocomitato studentesco, ASDA, fasc. Costantinopoli 1895-1921, 167A.

77 Cfr. GIBELLI, Antonio, Il popolo bambino. Infanzia e nazione dalla grande guerra a Salò, Torino, Einaudi, 2005.

78 Ibidem, p. 4. 
italiane presso circoli giovanili di altre nazioni79, ma l'attivismo del sodalizio andò declinando nel dopoguerra, come riferisce Luigi Joli a Roma:

\begin{abstract}
Il sottocomitato studentesco si ricompose con elementi non più disciplinati come prima. Gli ex scolari erano diventati giovinotti azzimati e boriosi, con pose d'impiegati superiori del Banco di Roma o della nuova banca commerciale o della nuovissima banca di Sconti. E la loro attività si svolse piuttosto a dar delle feste di ballo, e ne diedero un paio, devolvendo una buona parte degl'introiti ad offrire "champagne" e pasticcini alle signore e signorine civettuole. Il locale della Dante era diventato nient'altro che il ritrovo di questi neo-superuomini [...]. Fra i cortigiani v'era però qualche elemento possibile. Da questo fu presa l'iniziativa - a onor del vero - di ricominciare un corso d'italiano per le signorine affidandone l'incarico ad un certo sig. Porta, architetto, che faceva pure provvisoriamente l’insegnante alle RR. Scuole, aperte sì, ma pericolanti, perché il governo non mandava i maestri $[\ldots]^{80}$.
\end{abstract}

Rispetto a quanto stava avvenendo dal punto di vista educativo nel territorio nazionale, come si è già detto, la comunità italiana di Istanbul aveva fatto registrare un minimo ritardo, mancando, per esempio, esperienze centrali di aggregazione giovanile quali quella dello scoutismo, e neppure la guerra di Libia - che porterà la stessa Dante a maturare il definitivo passaggio dall'adesione agli ideali risorgimentali a quelli nazionalistici ${ }^{81}$ - aveva comportato cambiamenti significativi nell'organizzazione dell’infanzia e della gioventù italiana della colonia. Alla fine della grande guerra invece, anche la comunità italiana di Istanbul mostrò i segni della profonda cesura che il

79 «La gran maggioranza dei soci è costituita da giovanotti sudditi italiani; vi sono pure alcuni ottomani, elleni, tedeschi e austriaci. Il numero dei soci è dunque, chi consideri le condizioni della colonia e delle altre società italiane, ragguardevole. [...] Il sottocomitato si è dovuto contentare di iniziative modeste sia per l'esiguità delle proprie risorse, sia perché le iniziative più utili e più importanti erano già state assunte dal comitato maggiore, verso il quale i nostri sentimenti sono sempre stati deferentissimi e i nostri rapporti cordiali. Abbiamo voluto dotare di riviste italiane le sale di lettura dei vari circoli e clubs di qualsiasi nazionalità che ne erano privi e che si manifestarono disposti a gradirne l'invio. Le riviste scelte furono: Patria e Colonia, Lettura, Noi e il mondo. [Le riviste vennero spedite a varie associazioni giovanili] Young Man Christians Association; Union des employèes grecs de commerce; Società dalmata di beneficenza; Club grec; Unione italiana di Haidar-pascià; Club-Verein der deutschen juden [...]. Ma ci pareva doveroso che la maggior parte delle nostre risorse fosse quest'anno devoluta ad un atto che significasse la nostra devozione alla scuola nella quale la maggior parte dei nostri soci sono educati. Abbiamo provveduto ad acquistare e far inquadrare in eleganti e sobrie cornici riproduzioni in tricromia di insigni opere d'arte italiane. Sono dieci grandi quadri che, decoreranno la bella sala di disegno dei nostri regi istituti medi; e confidiamo che anche questo omaggio attesterà durevolmente quanto schiettamente noi siamo affezionati alla Scuola della Patria, e quanto il nostro stesso sodalizio si proponga di rinsaldare in ciascuno di noi questo sentimento", ASDA, fasc. Costantinopoli 1895-1921, 167A.

80 Joli a Zaccagnini, 11 gennaio 1920, ASDA, fasc. Costantinopoli 1895-1921, 167A.

${ }^{81}$ PISA, Beatrice, op. cit., pp. 285 sg. 
conflitto aveva impresso nelle popolazioni coinvolte. Anche se a livello strettamente societario il baricentro delle grandi discussioni del comitato centrale si era inevitabilmente spostato sull'azione dei comitati nelle terre irredente ${ }^{82}$, gli effetti più generali del conflitto non tardarono ad investire il Levante e uno dei primi segnali ad Istanbul fu proprio la riorganizzazione dei luoghi di sociabilità giovanile. Dell'indisciplina lamentata da Joli negli ambienti del sottocomitato studentesco non si ha più notizia, mentre lungo la grand Rue de Péra si impose il ritmo marziale delle parate dei Giovani esploratori che sfilavano nelle loro ordinate uniformi ${ }^{83}$, «ammiratissimi pel loro portamento corretto e disciplinato» ${ }^{84}$.

Il loro programma andava oramai oltre il generico attaccamento alla patria e all'amore per la lingua, «Il programma di vita dei "Giovani Esploratori” è un programma di rettitudine, di energia, di educazione morale e civile» ${ }^{85}$. Anche la Dante riprese le sue attività animata da nuovo spirito:

\begin{abstract}
Questo comitato locale ha deciso di tenere, fra qualche giorno, l'assemblea generale dei soci, che non aveva più avuto luogo dal principio della guerra, onde il nuovo consiglio che verrà eletto, possa riprendere l'attività dell'ante-guerra, e darsi con maggior lena alla propaganda dell'idea e della lingua italiana che devono imporsi in tutto il Levante, ora specialmente, che la dura guerra da noi vinta, ci ha innalzati alla vera funzione di grande potenza civilizzatrice nel mondo ${ }^{86}$
\end{abstract}

Nel luglio 1920, in un lunghissimo articolo intitolato L'Italia sul mare, ripercorrendo le antiche glorie italiche dalle imprese di Roma a quelle del Duca degli Abruzzi, si riproponeva il consueto topos del ritorno allo splendido passato, per poi chiudere con l'esortazione dannunziana «arma la prora e salpa verso il mondo!»87. L'autore dell'articolo era Padre Ferdinando Parri da Pesaro ${ }^{88}$, che sarebbe divenuto solo pochi mesi dopo uno dei più energici sostenitori del fascismo di Istanbul. Parri fu anche uno dei promotori, nel 1922, del segretariato cittadino dell'“Italica Gens"89, la

\footnotetext{
82 Ibidem, p. 355 et seq.

83 Sull'attività del corpo dei Giovani esploratori durante la guerra, riferimenti in VENTRONE, Angelo, La seduzione totalitaria. Guerra, modernità, violenza politica (1914-1918), Roma, Donzelli, 2003, p. 68; FERRETTI, Lando, Il libro dello sport, Roma, Libreria del Littorio, 1928.

84 Bollettino della Dante (da qui Bollettino), IV, 21/1923, p. 77.

85 I Giovani Esploratori, Trento, Tip. Art. Tridentina G. Moncher, 1916, p. 11.

86 Zeri alla presidenza centrale, ASDA, fasc. Costantinopoli 1895-1921, 167A.

87 Bollettino, I, 2/1920, p. 14.

88 Autore di un volume sulla missione francescana a Istanbul, PARRI, Ferdinando, Costantinopoli e i Francescani, Pesaro, Tip. Federici, 1930.

89 Sulla "Italica Gens" cfr. ROSOLI, Gianfausto, «La federazione "Italica Gens" e l'emigrazione italiana oltreoceano, 1909-1920», in Il Veltro, 24, 1-2/1990, pp. 87-100; TOMASI, Silvano M., «Fede e patria: the "Italica Gens" in the United States and Canada, 1908-1936. Notes for the
} 
Federazione per l'assistenza degli emigranti d'oltre oceano e del Levante. L'associazione, improntata a uno spirito patriottico e nazionale, faceva parte di quella costellazione di organismi sorti nel primo decennio del Novecento per rispondere all'esigenza della Chiesa di intervenire in chiave assistenziale nella questione migratoria.

Quanto ai rapporti fra la Dante e la Chiesa, a livello nazionale l'influente personalità di Ernesto Nathan, alla vicepresidenza della Dante dal 1889 al 1920, aveva mantenuto con fermezza una linea di laicità - seppure mai connotata da anticlericalismo - che a partire dal 1920 sarebbe venuta meno, denotando una maggiore apertura agli ambienti clericali9o. La prima adunanza dell'“Italica Gens” si tenne nella consueta sala della Società operaia, il primo dato evidente che emerge dal resoconto che propone il Bollettino è come i membri del comitato siano i soliti Joli, Mongeri, Pellegrini, Chabert, Leone (le stesse persone cioè che animavano e guidavano la Dante, la Società operaia, la Società di beneficienza e praticamente tutte le altre forme di associazionismo italiano della colonia). L'elemento di novità era costituito invece dalla presenza di Padre Parri, che tenne il discorso inaugurale e spiegò quale sarebbe stata l'azione del nuovo organismo - «essa, in perfetta intesa con le autorità governative, si propone di coordinare la sua attività a quella spiegata dallo Stato e di supplire colla sua organizzazione ai molti bisogni e ai molti compiti cui l'azione governativa non può arrivare» - quali le sue finalità - «migliorare le condizioni religiose, morali, intellettuali ed economiche degli italiani all'estero» - e quali i mezzi - «Chiesa, Scuola, Segretariato»91.

Dopo aver ribadito che l'Italica aveva un carattere eminentemente nazionale e sociale, e che non era un'associazione religiosa, Parri spiegava il motivo per cui la Chiesa aveva, di fatto, un ruolo essenziale come luogo dove «nel rito, nella lingua, nelle manifestazioni dell'arte trovino la Patria, mantengano il loro carattere e conservino il conforto di congiungere insieme i due grandi ideali: Dio e Patria». La morale umana, sosteneva Parri, doveva essere alla base di ogni azione, a prescindere dalle posizioni sociali, religiose o politiche degli individui, ma mentre una piccolissima parte dell'umanità poteva rintracciarla al di fuori della religione, nella scienza o nella filosofia, continuava il francescano, il popolo non conosceva queste distinzioni, dunque,

History of an Emigration Association», in Studi Emigrazione 28, 103, 1991, pp. 319-341; SARESELLA, Daniela, La lingua italiana nel mondo attraverso l'opera delle congregazioni religiose. Atti del convegno di studio, Perugia, 10 dicembre 1999, Soveria Mannelli, Rubbettino, 2001. Si vedano in particolare le pp. 51 et seq.

90 PISA, Beatrice, op. cit., pp. 398-399.

${ }^{11}$ Bollettino, III, 15/1922. 
concludeva, per il popolo moralità e religione coincidono, «insegnare al popolo la religione è insegnargli la base della morale, la sorgente dei suoi doveri, la ragione ultima della sua onestà, il segreto della sua grandezza»92. Altrettanto importante per la conservazione del sentimento nazionale era considerata la scuola, non solo in quanto luogo di apprendimento della lingua e della cultura natie, bensì anche come spazio di formazione di una sorta di coscienza dell'emigrato che veniva così affrancato dal ruolo che - nella visione dei nazionalisti - gli era stato attribuito dai liberali, quello cioè di protagonista in negativo di un'emigrazione che svuotava il paese delle sue forze vitali e produttive ${ }^{93}$. Il nuovo emigrante doveva essere invece investito in modo diretto e consapevole della sua novella missione imperialista:

\begin{abstract}
la Federazione si interessa perché si dia alla lingua italiana un’importanza uguale a quella della lingua del luogo, dove s'ispiri all'emigrato che il suo esilio non è effetto di decadenza della nostra Nazione, ma di fenomeni transitori economico-sociali. È questo un punto che L'“Italica Gens" vuole ben chiarito e sviluppato nelle scuole, affinché i giovani abbiano del fatto dell'emigrazione una comprensione esatta. Essi devono restar persuasi che certi fenomeni economico-sociali sono inevitabili nella vita di un popolo come il nostro di antica civiltà, ma di forze sempre giovani; di un popolo cui all'esuberanza dello sviluppo demografico sempre crescente, è venuto meno il relativo concomitante sviluppo economico; devono comprendere insomma che l'emigrazione, se considerata nei suoi effetti immediati e negli interessi individuali, può sembrare una triste e deplorevole necessità; considerata però negli effetti remoti e negli interessi nazionali, deve essere giudicata come mezzo di penetrazione italiana all'estero 94 .
\end{abstract}

Si preparavano così le basi di quella trasformazione dell'emigrazione che il fascismo, soprattutto attraverso l'azione di Dino Grandi, avrebbe formalizzato a partire dal 1926 nel passaggio da emigrati a italiani all'estero, un passaggio che non trovò impreparata la comunità di Istanbul 95 .

\footnotetext{
92 Ibidem.

93 Cfr. SORI, Ercole, L'emigrazione italiana dall'Unità alla seconda guerra mondiale, Bologna, Il Mulino, 1979.

94 Bollettino, III, 15, 1922.

95 Sulla politica estera del fascismo e la sua genesi fra la vasta bibliografia si vedano: COLLOTTI, Enzo (a cura di), Fascismo e politica di potenza. Politica estera 1922-1939, Scandicci, La Nuova Italia, 2000; GARZARELLI, Benedetta, Parleremo al mondo intero. La propaganda del fascismo all'estero, Alessandria, Edizioni dell'Orso, 2004; CAROCCI, Giampiero, La politica estera dell'Italia fascista (1925-1928), Roma-Bari, Laterza, 1969; RUMI, Giorgio, Alle origini della politica estera fascista (1918-1923), Roma-Bari, Laterza, 1968; DE FELICE, Renzo, L'Italia fra tedeschi e alleati. La politica estera fascista e la seconda guerra mondiale, Bologna, Il Mulino, 1973; PETRELLI, Matteo, Il fascismo e gli italiani all'estero, Bologna, Clueb, 2010;
} 
Infine, il segretariato della federazione si sarebbe occupato delle esigenze più materiali della popolazione, esso sarebbe stato aperto a tutti, «a qualsiasi partito o fede appartengano. La miseria non è né cattolica, né ortodossa, né israelita e non ha colore politico: essa è semplicemente miseria» ${ }^{6}$. Rispetto all'azione praticamente uguale che svolgeva già la società italiana di beneficenza - Padre Parri aveva comunque specificato che l'Italica Gens non si proponeva di sostituirsi alle istituzioni già esistenti, ma di integrarne l'opera in perfetto accordo - il segretariato si prefiggeva di porre un'attenzione particolare all'assistenza agli ex-combattenti97, questa nuova categoria di bisognosi che, seconda solo a quella dei caduti, in tutta Europa e non solo oramai meglio di qualunque altra incarnava l'eroismo e il sacrificio nazionale ${ }^{98}$. Alla sacralità della patria si affiancò in questo periodo la sacralità della madre, della donna, sancita già negli anni della guerra di Libia99 ma che a Istanbul si manifestò soprattutto nel primo dopoguerra, dove oramai le commemorazioni erano sempre più spesso affidate alla potenza simbolica ed evocativa delle donne, come nel caso delle cerimonia di consegna delle bandiere durante la festa dello Statuto, uno dei pochi riferimenti alle idee risorgimentali sopravvissuti alla bufera nazionalista, ma declinato anch'esso oramai all'etica del sacrificio, alla commemorazione non più dei confini dell'Italia del 1861 ma dei «desiderati confini» del 1919, che tali erano rimasti alla fine della guerra.

Con questo spirito, durante la festa del giugno 1921 la marchesa Garroni, moglie del regio ambasciatore, consegnò la bandiera d'Italia all'Associazione nazionale dei combattenti: "Al grido di guerra Voi, Italiani qui residenti, avete corrisposto accorrendo baldi e vigorosi col nome d'Italia sulle labbra, ma più ancora nel cuore, sotto la patria bandiera. [...] Onore a voi, o valorosi combattenti! A noi, donne d'Italia qui riunite, la soddisfazione di consegnarvi questo Vessillo che è segnacolo di valore in guerra e bandiera di civiltà in pace, di quella civiltà d'antica data che ha lasciato orme incancellabili in questo Oriente, che ci auguriamo veder ritornato a quella pace che è

CAVAROCCHI, Francesca, Avanguardie dello spirito. Il fascismo e la propaganda culturale all'estero, Roma, Carocci, 2010.

${ }_{96}$ Bollettino, III, 15, 1922.

97 Esisteva già in città una sezione locale dell'Associazione nazionale combattenti e reduci, fondata nel maggio 1921.

98 «Ricordiamolo sempre noi italiani, troppo avvezzi a dimenticare quello che di bene e di bello compimmo, dappoi che una turba dolorante di madri orbate dei loro figli, di vedove, di orfani, di giovani deturpati dalle ferite, passa ogi davanti ai nostri occhi ed attesta, come un quadro vivente di storia imperitura, il grande contributo dato all'Italia per il trionfo della causa comune [...], il doveroso tributo di onore a tutti quelli che caddero per un santo ideale», La festa del 4 novembre data in occasione del secondo anniversario della vittoria italiana, Bollettino, I, 4, 1920.

99 PISA, Beatrice, op. cit., pp. 279 et seq. 
nel desiderio di tutti» ${ }^{100}$. Un onore tanto più meritato in quanto «moltissimi di questi soldati non avevano mai visto l'Italia, che lo spirito mancava totalmente in questo paese e che parecchi non conoscevano neppure la lingua italiana!»101. Anche la raccolta di fondi per l'edificazione del monumento alla madre italiana riscosse grandi adesioni e, «con imponente solennità che non ha precedenti qui»102, in concomitanza con la tumulazione romana all'altare della patria, la colonia si raccolse nella giornata di commemorazione del milite ignoto, aperta dalla lunga prolusione del consueto Padre Parri.

Questo rigoglio di sentimento nazionale non poteva non ripercuotersi sulle attività più strettamente legate all'educazione dei giovai italiani di Istanbul. Il bollettino di agosto-settembre 1920 fu in gran parte dedicato al resoconto sul XXV congresso della Dante - il primo dopo l'interruzione del 1913 - che significativamente si svolse a Trieste, ma conteneva anche, oltre alle indicazioni sui soci e un articolo celebrativo per i caduti italiani nella guerra di Crimea, la cronaca della cerimonia di giuramento del corpo dei Giovani esploratori celebrata in concomitanza con le celebrazioni per il $50^{\circ}$ anniversario della presa di Roma, organizzata dalla Società Operaia. Come già accennato, a parte il sottocomitato studentesco della Dante, che attraversava una fase di declino, la nascita del corpo dei Giovani esploratori fu la prima esperienza di associazionismo organizzato del dopoguerra destinata alla gioventù stambuliota ${ }^{103}$. La cronaca della cerimonia di giuramento è un esempio concreto della forza dell'impatto della grande guerra sulla riorganizzazione delle strutture sociali, riprendendo il percorso già avviato a inizio secolo, la cura dell'educazione nazionale dei più giovani - o meglio, di educazione alla nazione - nel giro di pochissimi anni stravolse i suoi riferimenti, e in questo caso con maggiore evidenza, laddove i giovani italiani avevano l'arduo compito di rappresentare il corpo della nazione pur essendone, dimorando oltre i suoi confini, membra staccate:

${ }^{100}$ La festa dello "Statuto", Bollettino, II, 8, 1921.

101 Ibidem.

102 «Oggi, in Roma immortale, baciata dal sole autunnale, che indora i suoi monumenti, eterni come la sua bellezza, là, sull'altare della Patria che sovrasta l'Urbe, sull'altare della Patria simbolo magnifico della nostra unità indistruttibile, si è compiuta l'apoteosi dell'Eroe ignoto. [...] Questo figlio di tutte le madri è stato tumulato sull'altare sacro, mentre s'inchinavano davanti alle sue misere spoglie le mille bandiere lacere che sanno le battaglie [...] esso rappresenta il sacrifizio più sublime per il trionfo di un'idea, il sacrifizio della propria vita!». Ibidem.

${ }_{103}$ Il termine "stambuliota" è stato di recente oggetto di discussione e ritenuto preferibile, insieme a stanbuliota, rispetto alle varianti stambulino/stanbulino, pure utilizzate. Si coglie qui l'occasione per ringraziare Fabio L. Grassi per le preziose indicazioni in merito alla terminologia in lingua turca. 
I Giovani Esploratori avevano formato un largo quadrato, su due righe, al comando del capo-drappello di $2^{\circ}$ grado Tenente M. Pari. - Da un lato si erano schierate tutte le rappresentanze degli Esploratori stranieri, accorse numerose a festeggiare i compagni italiani. A un tratto, in mezzo a un silenzio imponente, il Tenente Pari s'avanza innanzi al gruppo delle Signore e delle Autorità riunite e stendendo la mano, pronunzia a voce chiara la formula del giuramento, a cui tutti gli esploratori rispondono col grido "lo giuro!". Un brivido di commozione percorre l'assistenza, mentre le musiche intonano la marcia reale. [...] Poi, la signora Arlotta, dominando l'emozione, impugna con virile baldanza la bandiera del corpo e dice ai giovani l'alto significato che deve avere per essi la bandiera che loro consegna, e aggiunge nobili parole, improntate di squisita gentilezza femminile. Infine la bandiera è consegnata al graduato Sig. Umberto Sogno. Gli esploratori sfilano in platea, con ordine perfetto, davanti alle Autorità ed alla folla plaudente ${ }^{104}$.

Il passo da questo tipo di cerimonia a quelle dei Balilla sarebbe stato brevissimo. La scarsa documentazione a disposizione non consente di sapere chi fossero i giovani esploratori, ma proprio sul Bollettino si legge che fin dalle sue prime settimane di esistenza si registrarono oltre duecento adesioni e che dalla comunità si raccolsero quarantamila lire italiane per provvedere alle esigenze di equipaggiamento. Le foto che corredano le cronache dei campi dei Giovani esploratori erano oramai immagini di soldati - «68 giovani italiani vissero per 20 giorni attendati, sotto il solleone di luglio ed a regime militare»105 - ragazzi in perfetta forma fisica ritratti nelle varie fasi del campo, "l'attendamento", "le esercitazioni sul mare", "il rancio", "il saluto alla bandiera", immortalati in pose atletiche e festose che ricordano lo spirito di agosto del 1914. Sublimati i morti della grande guerra attraverso la loro eroicizzazione, si preparava in questi anni una nuova generazione di combattenti e il nuovo nesso fra «guerra, gioventù e bellezza» ${ }^{106}$ era oramai riscontrabile anche a Istanbul, dove a questi presupposti si aggiunse la necessità di concorrere con i movimenti scoutistici di altra nazionalità sorti in città.

\footnotetext{
104 Bollettino, I, 3, 1920, p. 30.

105 Bollettino, III, 15, 1922.

${ }^{106}$ MOSSE, George, Sessualità e nazionalismo cit., pp. 129-151; si segnalano inoltre solo alcuni dei testi che maggiormente hanno approfondito questi aspetti fra la vastissima bibliografia a disposizione: ID. Le guerre mondiali. Dalla tragedia al mito dei caduti, Roma-Bari, Laterza, 2005, pp. 79-118 (per il mito del soldato caduto); FUSSEL, Paul, La grande guerra e la memoria moderna, Bologna, Il Mulino, 2000, pp. 345-394, GIBELLI, Antonio, La grande guerra e le trasformazioni del mondo mentale, Torino, Bollati Boringhieri, 1998. Per il periodo precedente si veda BONETTA, Gaetano, Corpo e nazione: l'educazione ginnastica, igienica e sessuale nell'Italia liberale, Milano, Franco Angeli, 1990.
} 
La Società nel frattempo continuò a promuovere le consuete iniziative. Sempre sotto la presidenza di Luigi Joli, nel novembre del 1920 i soci furono 802 ma già nel mese successivo si toccò quota 900, e il resoconto al 30 giugno 1921 riferisce di 1004 soci che sarebbero diventati 1122 a dicembre. Fu questo il quinquennio di maggiore crescita della Società, che avrebbe raggiunto i suoi massimi nel 1924 con 1252 soci. Nel 1921 ripresero i cicli di conferenze interrotti durante il conflitto, e anche in questo campo la profonda frattura rispetto ai temi dell'anteguerra fu evidente, come si deduce dal titolo della prima conferenza: L'aviazione come ausilio di guerra all'armata d'Italia $^{107}$. I corsi femminili continuarono a essere svolti e nel 1921 si sperimentò un corso serale misto per impiegati di banca e del commercio frequentato da circa 30 allievi. Nel 1922 i corsi per signore fecero registrare 55 iscritte, mentre in quelli serali gli allievi, che solitamente si attestavano sulla cinquantina, si ridussero della metà a causa dei licenziamenti legati al fallimento della Banca di sconto, che aveva messo ingentissimi capitali a disposizione delle scalate borsistiche dell'Ansaldo in quegli anni di profonda crisi legata alla riconversione postbellica delle industrie pesanti italiane ${ }^{108}$.

Ciò che non portò i risultati sperati fu il tentativo di avvicinare la popolazione studentesca turca allo studio della lingua italiana. Nel maggio 1921 dalla pubblicazione sul Bollettino di un elenco di nomi di allievi dei corsi serali segnalati per merito, si osserva una prevalenza di cognomi italiani, moltissimi ebraici di nazionalità non specificata e una piccolissima presenza di cognomi turchi. Nel 1922 il ministero degli affari esteri finanziò con diecimila lire corsi presso scuole turche e Joli, sfruttando la sua personale amicizia con il ministro ottomano all'istruzione, Said bey, riuscì ad istituire un corso di italiano nell'Istituto superiore del commercio di Istanbul e nella Scuola di belle arti. In quest'ultima, riferiva Joli, «già l'amico nostro arch. Mongeri è insegnante da circa quindici anni, spiegandovi un'ottima azione di propaganda per la cultura artistica italiana e lottando quotidianamente contro la potente e ben organizzata propaganda francese» ${ }^{109}$. Entrambi i corsi furono frequentati inizialmente da 22 allievi ciascuno,

ma dopo quattro o cinque lezioni, il prof. Colasanti rinunziò all'incarico, visto l'atteggiamento passivo, noncurante e velatamente ostile dei colleghi turchi, donde l'impressione che agisse di sottomano l'intrigo dei concorrenti. Tale atteggiamento si ripercoteva nella scolaresca, che si diradava ad ogni lezione. La questione era

\footnotetext{
107 Bollettino, II, 2, 1921-1922.

${ }^{108}$ LUPO, Salvatore, Il fascismo. La politica in un regime totalitario, Roma, Donzelli, 2005, p. 62.

109 Joli a Zaccagnini, 7 luglio 1922, ASDA, fasc. Costantinopoli 1895-1921, 167A.
} 
delicata ed occorreva prudenza, dato anche il momento alquanto difficile per lo stato d'animo degli ambienti turchi, mentre fra l'altro si stava inaugurando a Stanbul le nuove vie intitolate a Pierre Loti e a Claude Farrère! M’intesi dunque col direttore della scuola di commercio, Hulussi Bey, per sostituire il prof. Colasanti, "indisposto", col prof. Lacchia. [...] Il prof. Lacchia non ebbe miglior fortuna, ma seppe resistere sino alla fine, con vera abnegazione, di cui gli è dovuta ampia lode. Ed anche alla scuola di belle arti, ostacoli e difficoltà continue vennero ad intralciare la sua opera zelante ed assidua. Così, mentre fino a marzo le sue lezioni erano costantemente seguite da una quindicina di allievi, vennero aperti dei corsi liberi, di figura, con orario contemporaneo alle lezioni d'italiano. Nonostante le proteste fatte alla direzione, non fu possibile ottenere uno spostamento d'orario e molti alunni malgrado i richiami e gli sforzi per trattenerli, preferirono, ed era naturale, la lezione di figura che rappresentava per essi un interesse più diretto. Poi sorsero nuovi ostacoli, per causa di forza maggiore, come: "l'influenza", per cui tutte le scuole turche furono chiuse per una ventina di giorni; poi lo "sciopero degli studenti" delle scuole superiori, per il dissidio prodottosi fra essi ed i professori dell'Università, causa una malaugurata affermazione sostenuta da un insegnante, a proposito del celebre poeta nazionale Faissulì ${ }^{110}$, che sarebbe... persiano e non turco! Poi venne il "Ramazan", e poscia le feste di "Bairam", indi le frenetiche dimostrazioni in onore di Ferrére e finalmente le scuole turche si son chiuse per le vacanze estive! [...] Ad ogni modo, se questo nostro primo esperimento non ha prodotto, per cause di forza maggiore, l'esito sperato, noi siamo convinti della necessità di persistere a continuarlo per l'anno prossimo, interessando anzi il R. Governo a far pratiche dirette presso l'autorità ottomana, affinché i corsi di lingua italiana siano ufficialmente riconosciuti ed appoggiati nelle scuole turche e, almeno per il momento, in quelle scuole superiori a cui la Dante offre gl'insegnanti ${ }^{111}$.

Già trapelano fra queste righe le effervescenze che agitavano il paese alla vigilia della proclamazione della Repubblica. E proprio a partire dal 1923 si verificò un mutamento profondo nella vita del comitato, legato sia agli sviluppi della vita politica turca che di quella italiana. La proclamazione della repubblica di Turchia da una parte, dall'altra la fondazione di istituzioni fasciste in città, generarono inevitabilmente attrito fra due nazionalismi sempre più esuberanti e sempre meno propensi a condividere spazi destinati alla propaganda politica. Fra questi spazi, quello destinato

${ }^{110}$ Si tratta del poeta Fużūlī, nome di penna di Mehmed bin Süleyman (1483c.-1556), uno dei principali esponenti della poesia classica ottomana, «Fuzuli, Mehmed bin Süleyman», sub vocem in Encyclopædia Britannica, URL:

$<$ http://www.britannica.com/EBchecked/topic/222964/Mehmed-bin-Suleyman-Fuzuli > [consultato il 16 novembre 2014].

${ }_{111}$ Joli a Zaccagnini, 7 luglio 1922, ASDA, fasc. Costantinopoli 1895-1921, 167A. 
all'educazione dei giovani era considerato da entrambi i paesi un terreno eletto su cui coltivare il futuro delle due nazioni. 


\section{* L'autore}

Stefania De Nardis è dottore di ricerca in Storia contemporanea, collabora con la cattedra di Storia contemporanea dell'Università G. d'Annunzio di Chieti-Pescara e con la Fondazione per le scienze religiose Giovanni XXIII di Bologna.

URL: < http://www.studistorici.com/progett/autori/\#DeNardis >

\section{Per citare questo articolo:}

$\overline{\text { DE NARDIS, Stefania, «La Società Dante Alighieri da Costantinopoli a Istanbul. 1895-1922: diffusione della lingua } e}$ pedagogia nazionale», Diacronie. Studi di Storia Contemporanea : II diritto miltante, 29/12/2014,

URL:<http://www.studistorici.com/2014/12/29/de-nardis_numero_20/ >

\section{Diacronie Studi di Storia Contemporanea $\beta$ www.diacronie.it}

Risorsa digitale indipendente a carattere storiografico. Uscita trimestrale. redazione.diacronie@hotmail.it

Comitato di redazione: Jacopo Bassi - Luca Bufarale - Elisa Grandi - Deborah Paci - Fausto Pietrancosta - Matteo Tomasoni - Luca Zuccolo

Diritti: gli articoli di Diacronie. Studi di Storia Contemporanea sono pubblicati sotto licenza Creative Commons 2.5

(c) (7) $(-)$ Possono essere riprodotti a patto di non modificarne i contenuti e di non usarli per fini commerciali. La citazione di estratti è comunque sempre autorizzata, nei limiti previsti dalla legge. 